\title{
TRANSFER FUNCTIONS OF REGULAR LINEAR SYSTEMS. PART I: CHARACTERIZATIONS OF REGULARITY
}

\author{
GEORGE WEISS
}

\begin{abstract}
We recall the main facts about the representation of regular linear systems, essentially that they can be described by equations of the form $\dot{x}(t)=$ $A x(t)+B u(t), y(t)=C x(t)+D u(t)$, like finite dimensional systems, but now $A, B$ and $C$ are in general unbounded operators. Regular linear systems are a subclass of abstract linear systems. We define transfer functions of abstract linear systems via a generalization of a theorem of Fourés and Segal. We prove a formula for the transfer function of a regular linear system, which is similar to the formula in finite dimensions. The main result is a (simple to state but hard to prove) necessary and sufficient condition for an abstract linear system to be regular, in terms of its transfer function. Other conditions equivalent to regularity are also obtained. The main result is a consequence of a new Tauberian theorem, which is of independent interest.
\end{abstract}

\section{INTRODUCTION}

In the Hilbert space context, an abstract linear system is a linear system whose input, state and output spaces are Hilbert spaces, input and output functions are locally $L^{2}$, and on any finite time interval, the final state and the output function depend continuously on the initial state and the input function. The concept has been introduced by Salamon [15] to give a unifying framework for a large class of phenomena usually described as linear, time invariant and well posed. For the precise definition of this and other concepts used in the Introduction, see $\S 2$.

Regular linear systems are a large subclass of abstract linear systems, probably comprising every abstract linear system of practical interest. A system is regular if its output function corresponding to a step input function and zero initial state is not very discontinuous at zero; the precise requirement is that its average over $[0, \tau]$ should have a limit as $\tau \rightarrow 0$ (see $\S 2$ ). We often prefer to work with regular linear systems (as opposed to abstract linear systems) because they have a convenient representation, similar to that of finite dimensional systems, via equations (1.2) (for the precise statement see Theorem 2.3).

In this paper we investigate the transfer function of regular linear systems.

Received by the editors March 14, 1990 and, in revised form, August 20, 1992.

1991 Mathematics Subject Classification. Primary 93C25; Secondary 34G10, 44A10, 47D05.

Key words and phrases. Regular linear system, Lebesgue extension, shift-invariant operator, transfer function, feedthrough operator, Tauberian theorem.

This research was supported partially by the Weizmann Fellowship, and partially by the Air Force Office of Scientific Research under contract F49620-86-C-0111. 
We work in the Hilbert space context, but in remarks placed at the end of each section we make it clear which results extend to Banach spaces. Following is an outline of the paper, including the statement of some of the results.

In $\S 2$ we review the necessary background on abstract linear systems and regular linear systems. For easy reference, the proof of the representation theorem quoted in this section is given in the Appendix.

In $\S 3$ we give a generalization of a theorem of Fourés and Segal [9] concerning the representation of shift-invariant operators on $L^{2}$ by transfer functions in $H^{\infty}$. This is needed because the input/output map of an abstract linear system is a shift invariant operator on $L_{\text {loc }}^{2}$, not on $L^{2}$. We define the growth bound $\gamma_{0}$ of such an operator and prove that a transfer function representation is possible if and only if $\gamma_{0}<\infty$ (this condition is always satisfied by input/output maps of abstract linear systems).

The aim of $\S 4$ is to obtain a formula for the transfer function of a regular linear system. More precisely, we prove the following.

Theorem 1.1. Let $\Sigma$ be a regular linear system with semigroup generator $A$, control operator $B$, observation operator $C$, and feedthrough operator $D$. Then the transfer function $\mathbf{H}$ of $\Sigma$ is given for $s \in \mathbb{C}$ with sufficiently big real part by

$$
\mathbf{H}(s)=C_{L}(s I-A)^{-1} B+D,
$$

where $C_{L}$ is the Lebesgue extension of $C$.

The result has been announced (without proof) in Weiss [18, §4].

The first reaction of the reader to Theorem 1.1 might be to think that this is obvious. Indeed, we know (see $\S 2$ ) that $\Sigma$ is described by the following equations (which are satisfied for almost every $t \geq 0$ ):

$$
\begin{aligned}
& \dot{x}(t)=A x(t)+B u(t), \\
& y(t)=C_{L} x(t)+D u(t),
\end{aligned}
$$

where $u(\cdot)$ is the input function, of class $L_{\mathrm{loc}}^{2}, x(t)$ is the state at time $t$, and $y(\cdot)$ is the output function, again in $L_{\text {loc }}^{2}$. Applying formally the Laplace transformation to (1.2), we easily get (1.1). However, if we think more carefully, after applying the Laplace integral to equation (1.2b), we have to take $C_{L}$ out of the integral. But $C_{L}$ (like $C$ ) may be a nonclosable operator, so we have to be careful.

There are several ways of proving Theorem 1.1. We prefer to derive it as a consequence of another theorem which seems to be important in itself. This theorem gives a rather general answer to the question: when do $C_{L}$ and the integral sign commute? It turns out that this is true if $C_{L}$ is applied to a function $t \rightarrow \alpha(t) x(t)$, where $\alpha$ is scalar and $x$ is a state trajectory of $\Sigma$, and both functions do not grow too fast. For details see $\S 4$.

In $\S 5$ we first prove a new Tauberian theorem about the Laplace transformation of vector-valued functions of the class $L^{p}$.

Theorem 1.2. Let $W$ be a Banach space and $1<p<\infty$. Suppose $v \in$ $L^{p}([0, \infty), W)$ is such that

$$
\sup _{t>0} \frac{1}{t} \int_{0}^{t}\|v(\sigma)\|^{p} d \sigma<\infty .
$$


Let $\hat{v}$ denote the Laplace transform of $v$. Then, with $\lambda$ positive,

$$
\lim _{t \rightarrow 0} \frac{1}{t} \int_{0}^{t} v(\sigma) d \sigma=\lim _{\lambda \rightarrow \infty} \lambda \hat{v}(\lambda)
$$

i.e., if one of the limits exists, so does the other and they are equal.

We mention that if the limit on the left is known to exist then by a wellknown Abelian theorem (1.4) is true regardless if (1.3) holds. The interesting case is when only the limit on the right is known to exist. For more details and the proof see Proposition 5.1, Theorem 5.2, and Remark 5.9.

We need the above result in the proof of the spectral characterization of regularity, perhaps the main result of the paper, stated below (this is only a part of Theorem 5.8 in $\S 5$ ).

Theorem 1.3. An abstract linear system is regular if and only if its transfer function has a strong limit at $+\infty$ (along the real axis).

With the notation of Theorem 1.1, it is rather easy to prove that for any element $\mathrm{v}$ in the input space of $\Sigma$

$$
\lim _{\lambda \rightarrow \infty} \mathbf{H}(\lambda) \mathbf{v}=D \mathbf{v}
$$

where $\lambda$ is real. Theorem 1.3 shows that conversely, the existence of the limit in (1.5) implies that $\Sigma$ is regular. (Then it follows that $\Sigma$ has a feedthrough operator $D$, and $D$ can be computed by (1.5).)

A simple consequence of Theorem 1.3 is that the cascade connection of two regular systems is again regular. Maybe the most important consequence of Theorem 1.3 is that the class of regular linear systems is closed under feedback. However, we do not discuss cascades or feedback in this paper.

\section{ABSTRACT LINEAR SYSTEMS AND REgUlar LINEAR SYSTEMS: A REVIEW}

In this section we recall in a very sketchy way some definitions and results needed form other papers. Our aim is not to explain the material or to give proofs, only to fix the notation, to recall the main points of the theory and to indicate references.

First we introduce some notation. For any Hilbert space $W$ and any $\tau \geq$ $0, \mathbf{S}_{\tau}$ will denote the operator of right shift by $\tau$ on $L^{2}([0, \infty), W) . \quad \mathbf{P}_{\tau}$ will denote the projection of $L^{2}([0, \infty), W)$ onto $L^{2}([0, \tau], W)$ (by truncation), the latter space being regarded as a subspace of the former. For $u, v \in L^{2}([0, \infty), W)$ and $\tau \geq 0$, the $\tau$-concatenation of $u$ and $v$, denoted by $u \diamond v$, is defined by

$$
u \diamond v=\mathbf{P}_{\tau} u+\mathbf{S}_{\tau} v
$$

In other words, $\underset{\tau}{u \diamond} v)(t)=u(t)$ for $t \in[0, \tau)$, while $(\underset{\tau}{u \diamond} v)(t)=v(t-\tau)$ for $t \geq$ $\tau$. The shift and projection operators and the operation of concatenation have natural extensions to $L_{\text {loc }}^{2}([0, \infty), W)$, and we denote these extensions by the same symbols. We regard $L_{\text {loc }}^{2}([0, \infty), W)$ as a Fréchet space, with the topology given by the family of seminorms $p_{n}(u)=\left\|\mathbf{P}_{n} u\right\|_{L^{2}}$, so that $L^{2}([0, \infty), W)$ is dense in $L_{\mathrm{loc}}^{2}([0, \infty), W)$. 
Definition 2.1. Let $U, X$, and $Y$ be Hilbert spaces, $\Omega=L^{2}([0, \infty), U)$ and $\Gamma=L^{2}([0, \infty), Y)$. An abstract linear system on $\Omega, X$, and $\Gamma$ is a quadruple $\Sigma=(\mathbb{T}, \Phi, \Psi, \mathbb{F})$, where

(i) $\mathbb{T}=\left(\mathbb{T}_{t}\right)_{t \geq 0}$ is a strongly continuous semigroup of bounded linear operators on $X$,

(ii) $\Phi=\left(\Phi_{t}\right)_{t \geq 0}$ is a family of bounded linear operators from $\Omega$ to $X$ such that

$$
\Phi_{\tau+t}(u \underset{\tau}{\diamond} v)=\mathbb{T}_{t} \Phi_{\tau} u+\Phi_{t} v
$$

for any $u, v \in \Omega$ and any $\tau, t \geq 0$,

(iii) $\Psi=\left(\Psi_{t}\right)_{t \geq 0}$ is a family of bounded linear operators from $X$ to $\Gamma$ such that

$$
\Psi_{\tau+t} x=\Psi_{\tau} x \diamond_{\tau} \Psi_{t} \mathbb{T}_{\tau} x
$$

for any $x \in X$ and any $\tau, t \geq 0$, and $\Psi_{0}=0$,

(iv) $\mathbb{F}=\left(\mathbb{F}_{t}\right)_{t \geq 0}$ is a family of bounded linear operators from $\Omega$ to $\Gamma$ such that

$$
\mathbb{F}_{\tau+t}(u \diamond \tau)=\mathbb{F}_{\tau} u \diamond_{\tau}\left(\Psi_{t} \Phi_{\tau} u+\mathbb{F}_{t} v\right)
$$

for any $u, v \in \Omega$ and any $\tau, t \geq 0$, and $\mathbb{F}_{0}=0$.

$U$ is the input space of $\Sigma, X$ is the state space of $\Sigma$, and $Y$ is the output space of $\Sigma$. The operators $\Phi_{\tau}$ are called input maps. The operators $\Psi_{\tau}$ are called output maps. The operators $\mathbb{F}_{\tau}$ are called input/output maps.

Definition 2.1 has been reproduced from Weiss [18]. An equivalent definition has been given in Salamon [15]. The notation introduced in Definition 2.1 will be used throughout this section.

The intuitive meaning of the operators introduced above is the following: if $x(\tau)$ denotes the state of $\Sigma$ at time $\tau \geq 0$, and $u \in L_{\text {loc }}^{2}[(0, \infty), U)$ and $y \in L_{\mathrm{loc}}^{2}([0, \infty), Y)$ are the input and output functions of $\Sigma$ respectively, then

$$
\left(\begin{array}{c}
x(\tau) \\
\mathbf{P}_{\tau} y
\end{array}\right)=\left(\begin{array}{ll}
\mathbb{T}_{\tau} & \mathbf{\Phi}_{\tau} \\
\Psi_{\tau} & \mathbb{F}_{\tau}
\end{array}\right) \cdot\left(\begin{array}{c}
x(0) \\
\mathbf{P}_{\tau} u
\end{array}\right) .
$$

The truncated function $\mathbf{P}_{\tau} u$ belongs to the Hilbert space $L^{2}([0, \infty), U)$, and similarly for $\mathbf{P}_{\tau} y$, so that all operators above map Hilbert spaces into Hilbert spaces, as required in the definition.

The simplest example of an abstract linear system is a finite dimensional system described by the equations

$$
\dot{x}(t)=A x(t)+B u(t), \quad y(t)=C x(t)+D u(t),
$$

where $A, B, C$, and $D$ are matrices of appropriate dimensions. In this case, by a trivial computation

$$
\begin{gathered}
\mathbb{T}_{\tau}=e^{A \tau}, \\
\Phi_{\tau} u=\int_{0}^{\tau} e^{A(\tau-\sigma)} B u(\sigma) d \sigma, \\
\left(\Psi_{\tau} x\right)(t)=C e^{A t} x, \quad \text { for } t \in[0, \tau), \\
\left(\mathbb{F}_{\tau} u\right)(t)=C \int_{0}^{t} e^{A(t-\sigma)} B u(\sigma) d \sigma+D u(t), \quad \text { for } t \in[0, \tau) .
\end{gathered}
$$

For $t \geq \tau$ we have $\left(\Psi_{\tau} x\right)(t)=0$ and $\left(\mathbb{F}_{\tau} u\right)(t)=0$. 
It is surprising that for any abstract linear system, the operators $\mathbb{T}_{\tau}, \Phi_{\tau}$, and $\Psi_{\tau}$ can be expressed by formulae not much different from the above. For $\mathbb{F}_{\tau}$ this is true if an additional assumption is made, regularity. Before going into these matters, we give a more interesting but still very simple example.

Example. We model a delay line as an abstract linear system. Let $X=L^{2}[-h, 0]$, where $h>0$, and let $\mathbb{T}$ be the left shift semigroup on $X$ with zero entering from the right, i.e., for any $\tau \geq 0$ and $\zeta \in[-h, 0]$

$$
\left(\mathbb{T}_{\tau} x\right)(\zeta)= \begin{cases}x(\zeta+\tau), & \text { for } \zeta+\tau \leq 0 \\ 0, & \text { for } \zeta+\tau>0\end{cases}
$$

Let $U=\mathbb{C}$ and for any $\tau \geq 0$ and $\zeta \in[-h, 0]$

$$
\left(\Phi_{\tau} u\right)(\zeta)= \begin{cases}u(\zeta+\tau), & \text { for } \zeta+\tau \geq 0, \\ 0, & \text { for } \zeta+\tau<0 .\end{cases}
$$

Let $Y=\mathbb{C}$ and for any $\tau \geq 0$ and $t \in[0, \tau)$

$$
\left(\Psi_{\tau} x\right)(t)= \begin{cases}x(t-h), & \text { for } t-h \leq 0, \\ 0, & \text { for } t-h>0 .\end{cases}
$$

For $t \geq \tau$ we put $\left(\Psi_{\tau} x\right)(t)=0$. Finally, let for any $\tau \geq 0$ and $t \in[0, \tau)$

$$
\left(\mathbb{F}_{\tau} u\right)(t)= \begin{cases}u(t-h), & \text { for } t-h \geq 0, \\ 0, & \text { for } t-h<0 .\end{cases}
$$

For $t \geq \tau$ we put $\left(\mathbb{F}_{\tau} u\right)(t)=0$. Then $\Sigma=(\mathbb{T}, \Phi, \Psi, \mathbb{F})$ is an abstract linear system. For another example worked out in detail the reader may look up Curtain and Weiss [6].

From the definition of an abstract linear system given above, one can derive the following two formulae expressing causality:

$$
\Phi_{\tau} \mathbf{P}_{\tau}=\Phi_{\tau}, \quad \mathbb{F}_{\tau} \mathbf{P}_{\tau}=\mathbb{F}_{\tau},
$$

for any $\tau \geq 0$. It is also easy to show that for $0 \leq \tau \leq T$

$$
\mathbf{P}_{\tau} \Psi_{T}=\Psi_{\tau}, \quad \mathbf{P}_{\tau} \mathbb{F}_{T}=\mathbb{F}_{\tau} .
$$

Let $A$ denote the generator of $\mathbb{T}$. The Hilbert spaces $X_{1}$ and $X_{-1}$ are defined as follows: $X_{1}$ is $D(A)$ with the norm $\|x\|_{1}=\|(\beta I-A) x\|$, where $\beta \in \rho(A)$ is fixed (this is equivalent with the graph norm), and $X_{-1}$ is the completion of $X$ with respect to the norm $\|x\|_{-1}=\left\|(\beta I-A)^{-1} x\right\|$. These spaces are independent of the choice of $\beta$. If $D\left(A^{*}\right)$ is endowed with its graph norm then $X_{-1}$ can be identified with $D\left(A^{*}\right)^{*}$, the dual of $D\left(A^{*}\right)$ with respect to the scalar product of $X$. We have $X_{1} \subset X \subset X_{-1}$, densely and with continuous embeddings. The semigroup $\mathbb{T}$ can be restricted to a semigroup on $X_{1}$ and extended to a semigroup on $X_{-1}$. These three semigroups are isomorphic and we shall denote them by the same symbol (the isomorphism from $X$ to $X_{1}$ is $(\beta I-A)^{-1}$ and its extension to $X_{-1}$ is the isomorphism from $X_{-1}$ to $\left.X\right)$. The generator of $\mathbb{T}$ on $X_{1}$ is the restriction of $A$ to $D\left(A^{2}\right)$ and the generator of $\mathbb{T}$ on $X_{-1}$ is the extension of $A$ to $X$, which is bounded as an operator from $X$ to $X_{-1}$. Like in the case of $\mathbb{T}$, we will use the same notation for the original generator $A$ and for its restriction and extension described 
above. More information on the spaces $X_{1}$ and $X_{-1}$ can be found for example in Weiss [16].

By a representation theorem due to Salamon [15] (see also Weiss [6]), there is a (unique) $B \in \mathscr{L}\left(U, X_{-1}\right)$, called the control operator of $\Sigma$, such that for any $\tau \geq 0$

$$
\Phi_{\tau} u=\int_{0}^{\tau} \mathbb{T}_{\tau-\sigma} B u(\sigma) d \sigma .
$$

$B$ is called bounded if it belongs to $\mathscr{L}(U, X)$, and unbounded otherwise. $B$ is a strong limit of bounded control operators: if we put for any $\tau>0$

$$
B^{\tau} \mathrm{v}=\Phi_{\tau}(\chi \cdot \mathrm{v}) / \tau, \quad \forall \mathrm{v} \in U
$$

where $\chi$ is the constant function on $[0, \infty)$ equal to 1 everywhere, then $B \mathrm{v}=$ $\lim _{\tau \rightarrow 0} B^{\tau} \mathbf{v}$ (in $X_{-1}$ ) for any $\mathbf{v} \in U$, see Weiss [16, formula (3.6)].

For any $x_{0} \in X$ and $u \in L_{\text {loc }}^{2}([0, \infty), U)$, the function $x:[0, \infty) \rightarrow X$ defined by $x(t)=\mathbb{T}_{t} x_{0}+\Phi_{t} u$ (compare with (2.4)) is called the state trajectory of $\Sigma$ starting from $x_{0}$, corresponding to the input $u$. It follows from (2.5) that $x$ is a strong solution (in $X_{-1}$ ) of the differential equation

$$
\dot{x}(t)=A x(t)+B u(t),
$$

as defined in Weiss [16, Definition 3.5]. Equivalently, both sides are in $L_{\text {loc }}^{2}\left([0, \infty), X_{-1}\right)$ and are equal for a.e. $t \geq 0$. Moreover, $x$ is continuous from $[0, \infty)$ to $X$. For details see [16, §3].

We shall often need the Fréchet spaces

$$
\widetilde{\Omega}=L_{\text {loc }}^{2}([0, \infty), U), \quad \widetilde{\Gamma}=L_{\text {loc }}^{2}([0, \infty), Y)
$$

(see the notation at the beginning of this section). The operators $\mathbb{F}_{\tau}$ have natural continuous extensions to $\widetilde{\Omega}$. If we regard the operators $\Psi_{\tau}$ as elements of $\mathscr{L}(X, \widetilde{\Gamma})$, and the operators $\mathbb{F}_{\tau}$ as elements of $\mathscr{L}(\widetilde{\Omega}, \widetilde{\Gamma})$, then these operator families have strong limits as $\tau \rightarrow \infty$, denoted $\Psi_{\infty}$ and $\mathbb{F}_{\infty}$. These are convenient to work with, since for any $\tau \geq 0$, the operators $\Psi_{\tau}$ and $\mathbb{F}_{\tau}$ are obtained from them by truncation:

$$
\Psi_{\tau}=\mathbf{P}_{\tau} \Psi_{\infty}, \quad \mathbb{F}_{\tau}=\mathbf{P}_{\tau} \mathbb{F}_{\infty} .
$$

We shall call $\Psi_{\infty}$ the extended output map of $\Sigma$, and $\mathbb{F}_{\infty}$ the extended input/output map of $\Sigma$. Formulae (2.2) and (2.3) have the following extensions:

$$
\begin{gathered}
\Psi_{\infty} x=\Psi_{\infty} x \diamond_{\tau} \Psi_{\infty} \mathbb{T}_{\tau} x, \\
\mathbb{F}_{\infty}(u \stackrel{\diamond}{u} v)=\mathbb{F}_{\infty} u \diamond_{\tau}\left(\Psi_{\infty} \Phi_{\tau} u+\mathbb{F}_{\infty} v\right),
\end{gathered}
$$

for any $x \in X$, any $u, v \in \widetilde{\Omega}$ and any $\tau \geq 0$. For further details on these extended maps see Weiss $[17,18]$.

By a representation theorem due to Salamon [15] (see also Weiss [17]), there is a (unique) $C \in \mathscr{L}\left(X_{1}, Y\right)$, called the observation operator of $\Sigma$, such that for $x_{0} \in X_{1}$ and $t \geq 0$

$$
\left(\Psi_{\infty} x_{0}\right)(t)=C \mathbb{T}_{t} x_{0}
$$


$C$ is called bounded if it can be extended continuously to $X$, and unbounded otherwise. The above formula determines $\Psi_{\infty}$, because $X_{1}$ is dense in $X$. A simple but useful consequence of the above formula is that for any $x_{0} \in X$

$$
\int_{0}^{\tau}\left(\Psi_{\infty} x_{0}\right)(\sigma) d \sigma=C \int_{0}^{\tau} \mathbb{T}_{\sigma} x_{0} d \sigma
$$

The Lebesgue extension of $C$ is defined by

$$
C_{L} x_{0}=\lim _{\tau \rightarrow 0} C \frac{1}{\tau} \int_{0}^{\tau} \mathbb{T}_{\sigma} x_{0} d \sigma,
$$

with domain

$$
D\left(C_{L}\right)=\left\{x_{0} \in X \mid \text { the limit in (2.12) exists }\right\},
$$

which satisfies $X_{1} \subset D\left(C_{L}\right) \subset X$. For any $x_{0} \in X$ we have that $\mathbb{T}_{t} x_{0} \in D\left(C_{L}\right)$ for almost every $t \geq 0$ and

$$
\left(\Psi_{\infty} x_{0}\right)(t)=C_{L} \mathbb{T}_{t} x_{0}, \quad \text { for a.e. } t \geq 0 .
$$

For more information on $C_{L}$ see Weiss [17].

For any $\mathbf{v} \in U$, the function

$$
y_{v}=\mathbb{F}_{\infty}(\chi \cdot \mathbf{v})
$$

is the step response of $\Sigma$ corresponding to $\mathbf{v}$ ( $\chi$ was defined after (2.6)).

Definition 2.2. With the notation of Definition 2.1, $\Sigma$ is regular if for any $\mathbf{v} \in U$, the corresponding step response $y_{\mathbf{v}}$ has a Lebesgue point at 0 , i.e., the following limit exists (in $Y$ ):

$$
D \mathbf{v}=\lim _{\tau \rightarrow 0} \frac{1}{\tau} \int_{0}^{\tau} y_{\mathrm{v}}(\sigma) d \sigma .
$$

In that case, the operator $D \in \mathscr{L}(U, Y)$ defined by (2.14) is called the feedthrough operator of $\Sigma$.

The above definition has been reproduced from Weiss [18]. The concept of regular linear system has been vaguely anticipated by Helton [12] in his definition of "compatible systems" (see also Fuhrmann [10, p. 296]). A class of abstract linear systems which have received much attention in recent years are the "Pritchard-Salamon systems" (see Curtain et al. [5] for an updated theory and references). These systems have continuous step response and so they are regular. The delay line described in the Example is obviously regular, but it is not a Pritchard-Salamon system.

For regular linear systems we have the following representation theorem, which is the main result of the paper Weiss [18].

Theorem 2.3. Let $\Sigma=(\mathbb{T}, \Phi, \Psi, \mathbb{F})$ be a regular linear system, with input space $U$, state space $X$ and output space $Y$. Let $A$ be the generator of $\mathbb{T}, B$ the control operator of $\Sigma, C$ the observation operator of $\Sigma, C_{L}$ the Lebesgue extension of $C$, and $D$ the feedthrough operator of $\Sigma$.

Then for any $x_{0} \in X$ and any $u \in L_{\mathrm{loc}}^{2}([0, \infty), U)$, the functions $x:[0, \infty)$ $\rightarrow X$ and $y \in L_{\mathrm{loc}}^{2}([0, \infty), Y)$ defined by

$$
\begin{aligned}
x(t) & =\mathbb{T}_{t} x_{0}+\Phi_{t} u, \\
y & =\Psi_{\infty} x_{0}+\mathbb{F}_{\infty} u,
\end{aligned}
$$


satisfy equations (1.2) for a.e. $t \geq 0$ (in particular, $x(t) \in D\left(C_{L}\right)$ for a.e. $t \geq 0$ ). The function $x$ given by (2.15a) is the unique strong solution of (1.2a) which satisfies the initial condition $x(0)=x_{0}$.

The proof is given in the Appendix ( $\S 6$ ). For abstract linear systems (not necessarily regular) a similar theorem holds, but $(1.2 \mathrm{~b})$ is replaced by a more complicated equation (which does not contain $D$, since there is no $D$ ). We do not go into this here, but the reader may consult Salamon [15].

Taking in (2.15) $x_{0}=0$ and combining (1.2b) with (2.5), we get that for regular linear systems, $\mathbb{F}_{\infty}$ is given by the formula

$$
\left(\mathbb{F}_{\infty} u\right)(t)=C_{L} \int_{0}^{t} \mathbb{T}_{t-\sigma} B u(\sigma) d \sigma+D u(t),
$$

valid for any $u \in L_{\text {loc }}^{2}([0, \infty), U)$ and almost every $t \geq 0$ (in particular, the integral above belongs to $D\left(C_{L}\right)$ for a.e. $\left.t \geq 0\right)$.

For a survey of the material presented in this section see Curtain [4].

Remark 2.4. Everything written in this section remains valid, with some minor and obvious modifications, if we replace Hilbert spaces by Banach spaces and $L^{2}$ by $L^{p}$, with $1 \leq p<\infty$. (There is one small exception: if the state space $X$ is nonreflexive, then $X_{-1}$ cannot be identified with $D\left(A^{*}\right)^{*}$.) For $p=\infty$, the representation (2.5) (and hence also (2.16)) is no longer valid, see Weiss [19, Remark 3.7]. The representation (2.13) remains valid, $C$ is bounded and (1.2b) holds if $u$ has a.e. a right limit.

\section{SHIFT INVARIANT OPERATORS ON $L_{\text {loc }}^{2}$ AND THEIR REPRESENTATION BY TRANSFER FUNCTIONS}

Notation. Throughout this section, $U$ and $Y$ are complex Hilbert spaces and $\widetilde{\boldsymbol{\Omega}}$ and $\widetilde{\Gamma}$ are $L_{\text {loc }}^{2}$ type spaces, as introduced in (2.7). For any $\alpha \in[-\infty, \infty)$, $\mathbb{C}_{\alpha}$ will denote the right open half-plane in $\mathbb{C}$ delimited by $\alpha$ :

$$
\mathbb{C}_{\alpha}=\{s \in \mathbb{C} \mid \operatorname{Re} s>\alpha\} .
$$

We denote for any Hilbert space $W$ and any $v \in L_{\text {loc }}^{2}([0, \infty), W)$

$$
\alpha_{2}(v)=\inf \left\{\lambda \in \mathbb{R} \mid \int_{0}^{\infty} e^{-2 \lambda t}\|v(t)\|^{2} d t<\infty\right\} .
$$

In particular, for $v \in L^{2}([0, \infty), W)$ we have $\alpha_{2}(v) \leq 0$. It may happen that $\alpha_{2}(v)=\infty$. If $\alpha_{2}(v)<\infty$, then the Laplace transform $\hat{v}$ is defined for $s \in \mathbb{C}_{\alpha_{2}(v)}$ by

$$
\hat{v}(s)=\int_{0}^{\infty} e^{-s t} v(t) d t .
$$

(There are other definitions, yielding $\hat{v}$ defined on a possibly larger set, see e.g. Greiner et al. [11].) Recall the notations $\mathbf{P}_{\tau}$ and $\mathbf{S}_{\tau}$ for truncations and right shifts, introduced at the begining of $\S 2$.

An operator $\mathscr{F}: \widetilde{\Omega} \rightarrow \widetilde{\Gamma}$ is called shift invariant if

$$
\mathscr{F} \mathbf{S}_{\tau}=\mathbf{S}_{\tau} \mathscr{F}, \quad \forall \tau \geq 0 .
$$

Note that shift invariant operators are causal, i.e., $\mathbf{P}_{\tau} \mathscr{F}=\mathbf{P}_{\tau} \mathscr{F} \mathbf{P}_{\tau}$ holds for any $\tau \geq 0$. We are interested in shift invariant operators because for any abstract 
linear system, the extended input/output map $\mathbb{F}_{\infty}$ is shift invariant. Indeed, this follows from (2.9) by taking $u=0$.

We recall the following theorem of Fourés and Segal [9].

Theorem 3.1. Suppose $\mathscr{F}$ is a shift invariant bounded linear operator form $L^{2}([0, \infty), U)$ to $L^{2}([0, \infty), Y)$. Then there is a unique bounded analytic $\mathscr{L}(U, Y)$-valued function $\mathbf{H}$ defined on $\mathbb{C}_{0}$ such that for any $u \in$ $L^{2}([0, \infty), U)$, denoting $y=\mathscr{F} u$,

$$
\hat{y}(s)=\mathbf{H}(s) \hat{u}(s), \quad \forall s \in \mathbb{C}_{0} .
$$

Moreover,

$$
\sup _{s \in \mathbb{C}_{0}}\|\mathbf{H}(s)\|=\|\mathscr{F}\| .
$$

Conversely, any bounded analytic $\mathscr{L}(U, Y)$-valued function $\mathbf{H}$ defined on $\mathbb{C}_{0}$ defines via (3.1) a shift invariant bounded linear operator from $L^{2}([0, \infty), U)$ to $L^{2}([0, \infty), Y)$.

For an elementary proof, extensions and references see Weiss [19]. The function $\mathbf{H}$ appearing above is called the transfer function of $\mathscr{F}$.

Our goal is to extend Theorem 3.1 for shift invariant continuous operators from $\widetilde{\Omega}$ to $\widetilde{\Gamma}$. First we note that an extension is not always possible: indeed, consider $U=Y=\mathbb{C}$ and $\mathscr{F}$ given by

$$
(\mathscr{F} u)(t)=\int_{0}^{t} e^{(t-\sigma)^{2}} u(\sigma) d \sigma
$$

Then for any $u \neq 0$ with $u(t) \geq 0$, the Laplace transform of $\mathscr{F} u$ is nowhere defined. For $\mathscr{F}$ to be representable by a formula similar to (3.1), we need that for functions $u, y$ with $y=\mathscr{F} u$, the existence of $\hat{u}$ should imply the existence of $\hat{y}$. Since we agreed to define $\hat{u}$ on the half-plane delimited by $\alpha_{2}(u)$, and similarly for $\hat{y}$, this means that $\alpha_{2}(u)<\infty$ should imply $\alpha_{2}(y)<\infty$. Propositions 3.2 and 3.5 give several conditions on $\mathscr{F}$ that are equivalent to the one just stated.

We introduce some more notation. For any Hilbert space $W$ and any $\lambda \in \mathbb{R}$, the operator $e_{\lambda}$ on $L_{\text {loc }}^{2}([0, \infty), W)$ is defined by

$$
\left(e_{\lambda} v\right)(t)=e^{\lambda t} v(t), \quad \forall t \geq 0 .
$$

We denote

$$
L_{\lambda}^{2}([0, \infty), W)=e_{\lambda} L^{2}([0, \infty), W) .
$$

Often we omit the argument and simply write $L_{\lambda}^{2} . L^{2}$ is the same as $L_{0}^{2} . L_{\lambda}^{2}$ is a Hilbert space with the norm $\|v\|_{L_{\lambda}^{2}}=\left\|e_{-\lambda} v\right\|_{L^{2}}$. It is clear that $L_{\lambda_{1}}^{2} \subset L_{\lambda_{2}}^{2}$ for $\lambda_{1} \leq \lambda_{2}$, densely, and $\alpha_{2}(u)$ is the infimum of those $\lambda \in \mathbb{R}$ for which $u \in L_{\lambda}^{2}$.

Proposition 3.2. Suppose $\mathscr{F}$ is a shift invariant continuous linear operator from $\widetilde{\Omega}$ to $\widetilde{\Gamma}$ and $\gamma \in[-\infty, \infty)$. Then the following statements are equivalent.

(i) For some $\tau>0, u \in \mathbf{P}_{\tau} L^{2} \Rightarrow \alpha_{2}(\mathscr{F} u) \leq \gamma$.

(ii) For any $\tau \geq 0$, the implication in (i) holds.

(iii) For any $u \in \widetilde{\Omega}, \alpha_{2}(\mathscr{F} u) \leq \max \left\{\gamma, \alpha_{2}(u)\right\}$.

(iv) For any $\lambda>\gamma, \mathscr{F}$ restricts to a bounded linear operator $\mathscr{F}: L_{\lambda}^{2} \rightarrow L_{\lambda}^{2}$. 
Proof. The implications (iv) $\Rightarrow$ (iii) $\Rightarrow$ (ii) $\Rightarrow$ (i) are obvious or very easy. We close the loop by proving (i) $\Rightarrow$ (iv) .

Assume (i) holds and let $\lambda>\gamma$. Define the continuous linear operator $\mathscr{T}: \widetilde{\Omega} \rightarrow \widetilde{\Gamma}$ by

$$
\mathscr{T}=e_{-\lambda} \mathscr{F} e_{\lambda}
$$

It follows from the simple relation $e_{\alpha} \mathbf{S}_{t}=e^{\alpha t} \mathbf{S}_{t} e_{\alpha}$, valid for any real $\alpha$ and any $t \geq 0$, that $\mathscr{T}$ is shift invariant. Let $\varepsilon \in(0, \lambda-\gamma)$, then it follows from (i) that $\mathscr{T}$ maps $\mathbf{P}_{\tau} L^{2}$ into $L_{-\varepsilon}^{2}$. By the closed graph theorem, $\mathscr{T}$ is bounded from $\mathbf{P}_{\tau} L^{2}$ to $L_{-\varepsilon}^{2}$. Denote for $k \in\{0,1,2, \ldots\}$

$$
\mathscr{T}_{k}=\left(\mathbf{P}_{(k+1) \tau}-\mathbf{P}_{k \tau}\right) \mathscr{T} \mathbf{P}_{\tau} \text {. }
$$

$\mathscr{T}_{k}$ may be regarded as an operator from $L^{2}$ to $L^{2}$ and as such we estimate its norm. If we denote by $K$ the norm of $\mathscr{T} \mathbf{P}_{\tau}$ as an operator from $L^{2}$ to $L_{-\varepsilon}^{2}$ and take into account that the norm of $\mathbf{P}_{(k+1) \tau}-\mathbf{P}_{k \tau}$, as an operator from $L_{-\varepsilon}^{2}$ to $L^{2}$, is $e^{-k \tau \varepsilon}$, then it follows from the definition that

$$
\left\|\mathscr{T}_{k}\right\| \leq K e^{-k \tau \varepsilon}, \quad \forall k \in\{0,1,2, \ldots\} .
$$

Let $v \in L^{2}$ and $w=\mathscr{T} v$. Then $v$ and $w$ can be written in the form

$$
v=\sum_{j=0}^{\infty} \mathbf{S}_{j \tau} v_{j}, \quad w=\sum_{k=0}^{\infty} \mathbf{S}_{k \tau} w_{k},
$$

with $v_{j} \in \mathbf{P}_{\tau} L^{2}$ and $w_{k} \in \mathbf{P}_{\tau} L^{2}$. Indeed, $v_{j}=\mathbf{P}_{\tau} \mathbf{S}_{j \tau}^{*} v$, where $\mathbf{S}_{t}^{*}$ denotes left shift by $t$, and similarly for $w_{k}$. By a simple argument we have

$$
\mathbf{S}_{k \tau} w_{k}=\sum_{j=0}^{k} \mathscr{T}_{k-j} v_{j},
$$

so the sequence $\left(\left\|w_{k}\right\|\right)$ is bounded by the convolution of the sequences $\left(\left\|\mathscr{T}_{k}\right\|\right)$ and $\left(\left\|v_{j}\right\|\right)$. Let $M=\left\|\mathscr{T}_{0}\right\|+\left\|\mathscr{T}_{1}\right\|+\cdots$, which is finite according to (3.4). Since $\|v\|_{L^{2}}^{2}=\left\|v_{0}\right\|^{2}+\left\|v_{1}\right\|^{2}+\cdots$, and similarly for $w$, it follows that $\|w\|_{L^{2}} \leq$ $M\|v\|_{L^{2}}$, i.e., $\mathscr{T}$ is bounded from $L^{2}$ to $L^{2}$. Since $\mathscr{F}=e_{\lambda} \mathscr{T} e_{-\lambda}, \mathscr{F}$ is bounded from $L_{\lambda}^{2}$ to $L_{\lambda}^{2}$.

It is easy to see that the set of numbers $\gamma \in[-\infty, \infty)$ for which the equivalent conditions in Proposition 3.2 are satisfied is either empty or of the form $\left[\gamma_{0}, \infty\right)$, with $\gamma_{0} \in[-\infty, \infty)$.

Definition 3.3. Let $\mathscr{F}$ be a shift invariant continuous linear operator from $\widetilde{\Omega}$ to $\widetilde{\Gamma}$. Let $\gamma_{0}$ be the smallest element in $[-\infty, \infty)$ for which one (and hence any) of the conditions in Proposition 3.2 is satisfied. If there are no such numbers then we put $\gamma_{0}=\infty$. Then $\gamma_{0}$ is the growth bound of $\mathscr{F}$. We say that $\mathscr{F}$ is exponentially stable if $\gamma_{0}<0$.

It is possible to have $\gamma_{0}=-\infty$ for nonzero $\mathscr{F}$, for example if $\mathscr{F}$ is given by convolution with a measure having bounded support. In general, $\mathscr{F}$ does not restrict to a bounded linear operator from $L_{\gamma_{0}}^{2}$ to $L_{\gamma_{0}}^{2}$. For example, consider $U=Y=\mathbb{C}$ and $(\mathscr{F} u)(t)=\int_{0}^{t} u(\sigma) d \sigma$. Then $\gamma_{0}=0$, but $\mathscr{F}$ does not map $L^{2}$ into itself. We shall see in $\S 4$ that if $\mathscr{F}$ is the extended input/output map of 
an abstract linear system, and if $\omega_{0}$ is the growth bound of the corresponding semigroup, then $\gamma_{0} \leq \omega_{0}$.

The following lemma is some sort of uniform boundedness principle.

Lemma 3.4. Let $W$ be a Hilbert space and $\mathscr{T}$ a continuous linear operator from $W$ to $\widetilde{\Gamma}$. If for any $w \in W$ we have $\alpha_{2}(\mathscr{T} w)<\infty$, then there is a $\gamma \in[-\infty, \infty)$ such that for any $w \in W$ we have $\alpha_{2}(\mathscr{T} w) \leq \gamma$.

Proof. Denote for any $k, m \in \mathbb{N}$

$$
V_{k, m}=\left\{w \in W \mid\|\mathscr{T} w\|_{L_{k}^{2}} \leq m\right\} .
$$

It is not difficult to see that $V_{k, m}$ is closed in $W$. Moreover, the union of all the sets $V_{k, m}$ is $W$. By Baire's theorem, there exist $\gamma, \mu \in \mathbb{N}$ such that $V_{\gamma, \mu}$ has nonempty interior. Then $V_{\gamma, \mu}-V_{\gamma, \mu}$ contains a ball of radius $r>0$ centered at 0 . Since $V_{\gamma, \mu}-V_{\gamma, \mu} \subset V_{\gamma, 2 \mu}$, we get that for any $w \in W$ with $\|w\|<r$ we have $\|\mathscr{T} w\|_{L_{\gamma}^{2}} \leq 2 \mu$. Hence, for any $w \in W$ we have $\|\mathscr{T} w\|_{L_{\gamma}^{2}}<\infty$, i.e., $\alpha_{2}(\mathscr{T} w) \leq \gamma$.

Note. The author is indebted to Hans Zwart for the above short proof.

The number $\gamma$ which we constructed in the proof of Lemma 3.4 is positive, but of course that does not preclude the possibility of $\gamma<0$ or even $\gamma=-\infty$ being a valid choice for some operators $\mathscr{T}$.

Before stating the next proposition we note that if $\tau>0$ and $u \in \mathbf{P}_{\tau} L^{2}$, then $\alpha_{2}(u)=-\infty$.

Proposition 3.5. Suppose $\mathscr{F}$ is a shift invariant continuous linear operator from $\widetilde{\Omega}$ to $\widetilde{\Gamma}$, and let $\gamma_{0}$ be its growth bound. Then the following statements are equivalent.

(i) For some $\tau>0, u \in \mathbf{P}_{\tau} L^{2} \Rightarrow \alpha_{2}(\mathscr{F} u)<\infty$.

(ii) For any $u \in \widetilde{\Omega}, \alpha_{2}(u)<\infty \Rightarrow \alpha_{2}(\mathscr{F} u)<\infty$.

(iii) $\gamma_{0}<\infty$.

Proof. The implications (iii) $\Rightarrow($ ii) $\Rightarrow$ (i) are clear (look at (iii) of Proposition 3.2). The implication (i) $\Rightarrow$ (iii) follows from Lemma 3.4; taking $W=\mathbf{P}_{\tau} L^{2}$ (use (i) of Proposition 3.2 to define $\gamma_{0}$ ).

The above proposition shows that a generalization of Theorem 3.1 is conceivable only for operators with growth bound $<\infty$. Now we can state this generalization.

Theorem 3.6. Suppose $\mathscr{F}$ is a shift invariant continuous linear operator from $\widetilde{\Omega}$ to $\widetilde{\Gamma}$, with growth bound $\gamma_{0}<\infty$. Then there is a unique $\mathscr{L}(U, Y)$-valued function $\mathbf{H}$ defined on $\mathbb{C}_{\gamma_{0}}$, which satisfies the following.

(i) $\mathbf{H}$ is analytic and for any $\gamma>\gamma_{0}$ it is bounded on $\mathbb{C}_{\gamma}$, more precisely

$$
\sup _{s \in \mathbb{C}_{\gamma}}\|\mathbf{H}(s)\|=\|\mathscr{F}\|_{\mathscr{L}\left(L_{\gamma}^{2}, L_{\gamma}^{2}\right)} \cdot
$$

(ii) If $u \in \widetilde{\Omega}$ with $\alpha_{2}(u)<\infty$, then denoting $y=\mathscr{F} u$ and $\alpha_{0}=$ $\max \left\{\gamma_{0}, \alpha_{2}(u)\right\}$, we have

$$
\hat{y}(s)=\mathbf{H}(s) \cdot \hat{u}(s), \quad \forall s \in \mathbb{C}_{\alpha_{0}} .
$$


Conversely, suppose $\mathbf{H}$ is an $\mathscr{L}(U, Y)$-valued function on $\mathbb{C}_{\gamma_{1}}$, where $\gamma_{1} \in$ $[-\infty, \infty)$. If $\mathbf{H}$ is analytic and for any $\gamma>\gamma_{1}$, it is bounded on $\mathbb{C}_{\gamma}$, then $\mathbf{H}$ defines via (3.6) a shift invariant continuous linear operator from $\widetilde{\Omega}$ to $\widetilde{\Gamma}$, with growth bound $\gamma_{0} \leq \gamma_{1}$ (in particular, $\mathbf{H}$ has an extension to $\mathbb{C}_{\gamma_{0}}$ with properties (i) and (ii) above).

Proof. Let $\mathscr{F}$ and $\gamma_{0}$ be as in the first part of the theorem. For any $\gamma>\gamma_{0}$, put $\mathscr{T}_{\gamma}=e_{-\gamma} \mathscr{F} e_{\gamma}$, so $\mathscr{T}_{\gamma}$ is shift invariant (see the beginning of the proof of Proposition 3.2). By (iv) of Proposition 3.2, $\mathscr{F}$ is bounded from $L_{\gamma}^{2}$ to $L_{\gamma}^{2}$, so $\mathscr{T}_{\gamma}$ is bounded from $L^{2}$ to $L^{2}$. Moreover, since $e_{\gamma}$ is a norm preserving transformation from $L^{2}$ to $L_{\gamma}^{2}$, we have

$$
\left\|\mathscr{T}_{\gamma}\right\|_{\mathscr{L}\left(L^{2}, L^{2}\right)}=\|\mathscr{F}\|_{\mathscr{L}\left(L_{\gamma}^{2}, L_{\gamma}^{2}\right)} \text {. }
$$

By Theorem 3.1 there is a unique bounded analytic $\mathscr{L}(U, Y)$-valued function $\mathbf{G}_{\gamma}$ on $\mathbb{C}_{0}$, representing $\mathscr{T}_{\gamma}$ like in (3.1). Coming back to $\mathscr{F}$, we get that for any $u \in L_{\gamma}^{2}$, (3.6) holds with $\mathbf{H}_{\gamma}(s)=\mathbf{G}_{\gamma}(s-\gamma)$ in place of $\mathbf{H}(s)$. Moreover, by (3.2) and (3.7) we get that (3.5) holds in $\mathbf{H}_{\gamma}$ in place of $\mathbf{H}$.

Let $\left(\gamma_{n}\right)$ be a decreasing sequence in $\left(\gamma_{0}, \infty\right)$ such that $\gamma_{n} \rightarrow \gamma_{0} . \mathbf{H}_{\gamma_{n}}$ is defined on $\mathbb{C}_{\gamma_{n}}$, and it is analytic and bounded there. Since $L_{\gamma_{n+1}}^{2} \subset L_{\gamma_{n}}^{2}$ densely, $\mathbf{H}_{\gamma_{n}}$ is uniquely determined by the restriction of $\mathscr{F}$ to $L_{\gamma_{n+1}}^{2}$, which implies that $\mathbf{H}_{\gamma_{n}}$ is the restriction of $\mathbf{H}_{\gamma_{n+1}}$ to $\mathbb{C}_{\gamma_{n}}$. This makes it possible to define by induction an analytic function $\mathbf{H}$ on $\mathbb{C}_{\gamma_{0}}$ such that for any $n \in \mathbb{N}, \mathbf{H}_{\gamma_{n}}$ is a restriction of $\mathbf{H}$. It follows that for any $\gamma>\gamma_{0}, \mathbf{H}_{\gamma}$ is the restriction of $\mathbf{H}$ to $\mathbb{C}_{\gamma}$, so (i) and (ii) are satisfied.

Conversely, let $\mathbf{H}$ and $\gamma_{1}$ be as in the second part of the theorem. Let $\gamma>\gamma_{1}$ and $\mathbf{G}(s)=\mathbf{H}(s+\gamma)$. Then by the second part of Theorem 3.1, $\mathbf{G}$ defines via (3.1) a shift invariant bounded linear operator $\mathscr{T}$ from $L^{2}$ to $L^{2}$. Put $\mathscr{F}=e_{\gamma} \mathscr{T} e_{-\gamma}$, so $\mathscr{F}$ is shift invariant and bounded from $L_{\gamma}^{2}$ to $L_{\gamma}^{2}$, and for $u \in L_{\gamma}^{2}$ it satisfies (3.6) on $\mathbb{C}_{\gamma}$. Obviously $\mathscr{F}$ extends to a shift invariant continuous operator from $\widetilde{\Omega}$ to $\widetilde{\Gamma}$ and its growth bound $\gamma_{0}<\gamma$. This being true for any $\gamma>\gamma_{1}$, we get $\gamma_{0} \leq \gamma_{1}$.

Definition 3.7. When $\mathscr{F}$ and $\mathbf{H}$ are as in the first part of the above theorem, then $\mathbf{H}$ is called the transfer function of $\mathscr{F}$.

Remark 3.8. Almost everything written in this section remains valid, after some simple and obvious modifications, if we replace Hilbert spaces by Banach spaces and $L^{2}$ by $L^{p}$, with $1 \leq p<\infty$ (in particular, the notation $\alpha_{2}(v)$ should be replaced by $\left.\alpha_{p}(v)\right)$. The exceptions are the following: in (3.2) and (3.5), the sign $=$ has to be replaced by $\leq$, and the converse (last) parts of both Theorems 3.1 and 3.6 will no longer hold. This is because the converse part of Theorem 3.1 is a consequence of the Paley-Wiener theorem, which has no correspondent for Banach spaces. The proof of the first part of Theorem 3.1 for Banach spaces and $L^{p}$ can be found in Weiss [19]. For $p=\infty$, nothing remains true of Theorem 3.1, see Weiss [19] (Propositions 3.2 and 3.5 and Lemma 3.4 can be extended also for this case).

4. When CAN We take $C_{L}$ OUt OF AN INTEGRAL, AND THE FORMULA FOR THE TRANSFER FUNCTION

Recall that the growth bound of a strongly continuous semigroup $\mathbb{T}$ is the smallest element $\omega_{0} \in[-\infty, \infty)$ such that for any $\omega>\omega_{0}$, there is an $M_{\omega} \geq 1$ 
for which

$$
\left\|\mathbb{T}_{t}\right\| \leq M_{\omega} e^{\omega t}, \quad \forall t \geq 0 .
$$

In particular, $\mathbb{T}$ is exponentially stable if $\omega_{0}<0$.

As mentioned at the beginning of $\S 3$, the extended input/output map of any abstract linear system is a shift invariant continuous operator. We start by a simple proposition relating the growth bound of this operator (see Definition 3.3) with the growth bound of the semigroup. This proposition is due to Salamon [15, Lemma 2.1] but for greater clarity we also give a proof.

Proposition 4.1. Let $\Sigma=(\mathbb{T}, \Phi, \Psi, \mathbb{F})$ be an abstract linear system. Denote by $\omega_{0}$ of the growth bound of $\mathbb{T}$ and by $\gamma_{0}$ the growth bound of $\mathbb{F}_{\infty}$. Then

Proof. First we prove that

$$
\gamma_{0} \leq \omega_{0}
$$

$$
\alpha_{2}\left(\Psi_{\infty} x_{0}\right) \leq \omega_{0}, \quad \forall x_{0} \in X
$$

(the notation $\alpha_{2}(v)$ was introduced at the beginning of $\S 3$ ). We have for any $\lambda \in \mathbb{R}$ and any $n \in \mathbb{N}$

$$
\left\|\Psi_{n} x_{0}\right\|_{L_{\lambda}^{2}}=\left(\sum_{k=0}^{n-1} e^{-2 k \lambda}\left\|\Psi_{1} \mathbb{T}_{k} x_{0}\right\|_{L_{\lambda}^{2}}^{2}\right)^{1 / 2}
$$

(proof by induction using (2.2)). It follows that

$$
\left\|\Psi_{n} x_{0}\right\|_{L_{\lambda}^{2}} \leq M\left(\sum_{k=0}^{n-1} e^{-2 k \lambda}\left\|\mathbb{T}_{k}\right\|^{2}\right)^{1 / 2}\left\|x_{0}\right\|,
$$

where $M$ is the norm of $\Psi_{1}$ as an operator from $X$ to $L_{\lambda}^{2}$. Now let us assume that $\lambda>\omega_{0}$ and let $\omega \in\left(\omega_{0}, \lambda\right)$. Using the last inequality together with (4.1), we get that the operators $\Psi_{n}$ form $X$ to $L_{\lambda}^{2}$ are uniformly bounded. Since $\Psi_{n}=\mathbf{P}_{n} \Psi_{\infty}$, it follows that $\Psi_{\infty}$ is bounded from $X$ to $L_{\lambda}^{2}$. This being true for any $\lambda>\omega_{0}$, we have proved (4.2).

Choose $\tau>0$. For any $u \in \mathbf{P}_{\tau} L^{2},(2.9)$ implies

$$
\mathbb{F}_{\infty} u=\mathbb{F}_{\tau} u \diamond \Psi_{\tau} \Psi_{\infty} \Phi_{\tau} u
$$

Taking $x_{0}=\Phi_{\tau} u$ in (4.2), it follows that $\alpha_{2}\left(\mathbb{F}_{\infty} u\right) \leq \omega_{0}$. Using (i) of Proposition 3.2 to define $\gamma_{0}$, we get $\gamma_{0} \leq \omega_{0}$.

It follows from Proposition 4.1, together with Theorem 3.6, that $\mathbb{F}_{\infty}$ can be represented by means of a transfer function (defined on $\mathbb{C}_{\gamma_{0}}$ ). By the transfer function of $\Sigma$ we simply mean the transfer function of $\mathbb{F}_{\infty}$. The main aim of this section is to prove formula (1.1) expressing the transfer function of a regular linear system in terms of the operators appearing in the representation (1.2) (see Theorem 4.7). As noted in the Introduction, we prove this formula using a theorem concerning integrals containing $C_{L}$ (Theorem 4.6), which is of independent interest.

Remark 4.2. In Salamon [14, 15] and in Curtain and Weiss [6], the transfer function $\mathbf{H}$ of an abstract linear system was introduced in a different way, 
leading to $\mathbf{H}$ being defined on $\rho(A)$ (the resolvent set of the generator). I think that this is not natural, because the transfer function should depend only on the input/output map, and not on any particular state space realization attached to it. However, the transfer functions defined in these two approaches are equal on the half-plane $\mathbb{C}_{\omega_{0}}$, so that they determine the same input/output maps.

Remark 4.3. An immediate consequence of Proposition 4.1 is that exponential stability of $\mathbb{T}\left(\omega_{0}<0\right)$ implies exponential stability of $\mathbb{F}_{\infty}\left(\gamma_{0}<0\right)$. Several authors have investigated the following problem: under which additional assumptions on $\Sigma$ does the converse implication hold. Clearly this is equivalent to asking when $\gamma_{0}=\omega_{0}$ holds. We refer to Curtain [3], Curtain et al. [5, §5] and, for the most general results currently available, to Rebarber [13].

Notation. For the rest of this section we shall work with regular linear systems, using the notation introduced in (2.7), Theorem 2.3, and (3.3). This means that the symbols $U, X, Y$ (Hilbert spaces), $\widetilde{\Omega}, \widetilde{\Gamma}, L_{\omega}^{2}$ (function spaces), $\Sigma, \mathbb{T}, \Phi$, $\Psi, \mathbb{F}$ (regular linear system and its operator families) and $A, B, C, C_{L}, D$ (operators representing the system via (1.2)) need to further explanation. $\omega_{0}$ and $\gamma_{0}$ will be as in Proposition 4.1 (growth bounds). $\mathbf{P}_{\tau}$ and $\mathbf{S}_{\tau}$ will denote truncations and right shifts (see the beginning of $\S 2$ ). $\mathbf{S}_{\tau}^{*}$ will denote the operator of left shift by $\tau \geq 0$ on both $\widetilde{\Omega}$ and $\widetilde{\Gamma}$ (the restriction of $\mathbf{S}_{\tau}^{*}$ to $L^{2}$ is the adjoint of the restriction of $\mathbf{S}_{\tau}$ to $\left.L^{2}\right)$.

For any $\tau>0$, the operator $C_{L}^{\tau} \in \mathscr{L}(X, Y)$ is defined by

$$
C_{L}^{\tau} z=C \frac{1}{\tau} \int_{0}^{\tau} \mathbb{T}_{\sigma} z d \sigma
$$

It is clear from the definition that for any $z \in D\left(C_{L}\right), \lim _{\tau \rightarrow 0} C_{L}^{\tau} z=C_{L} z$.

It is an immediate consequence of Theorem 2.3 that if $x$ is a state trajectory of $\Sigma$ (see $\S 2)$, then $\lim _{\tau \rightarrow 0} C_{L}^{\tau} x(t)=C_{L} x(t)$ for a.e. $t \geq 0$. We prove that the convergence is valid also in another sense.

Lemma 4.4. Let $\omega>\omega_{0}$ and $u \in L_{\omega}^{2}([0, \infty), U)$. Let $x$ be a state trajectory of $\Sigma$ corresponding to the input $u$. Then for $C_{L}^{\tau}$ defined as in (4.3) we have

$$
\lim _{\tau \rightarrow 0} C_{L}^{\tau} x=C_{L} x \text { in } L_{\omega}^{2}([0, \infty), Y) .
$$

Proof. Let $\chi$ denote the constant function on $[0, \infty)$ equal to 1 everywhere. We define for every $\tau>0$ the operator $D^{\tau} \in \mathscr{L}(U, Y)$ by

$$
D^{\tau} \mathrm{v}=\frac{1}{\tau} \int_{0}^{\tau}\left(\mathbb{F}_{\infty}(\chi \cdot \mathrm{v})\right)(\sigma) d \sigma
$$

By (2.14), $\lim _{\tau \rightarrow 0} D^{\tau} \mathrm{v}=D \mathrm{v}$, so by the uniform boundedness principle the operators $D^{\tau}$ are uniformly bounded for $\tau \in(0,1]$. It follows by the Lebesgue dominated convergence theorem that

$$
\lim _{\tau \rightarrow 0} D^{\tau} u=D u \text { in } L_{\omega}^{2} .
$$

Let us derive a useful identity. Clearly for any $q, r \in \widetilde{\Gamma}$ and any $t \geq 0$, $\mathrm{S}_{t}^{*}(\underset{\tau}{\stackrel{\leftrightarrow}{\diamond} r)}=r$. Applying this to (2.8) and (2.9), we get that for any $z \in X$ and any $f, g \in \widetilde{\Omega}$,

$$
\mathbf{S}_{t}^{*} \Psi_{\infty} z=\Psi_{\infty} \mathbb{T}_{t} z
$$




$$
\mathbf{S}_{t}^{*} \mathbb{F}_{\infty}(f \underset{\tau}{\diamond} g)=\Psi_{\infty} \Phi_{t} f+\mathbb{F}_{\infty} g
$$

Adding these equalities and using that $f=f_{\tau}^{\diamond} \mathbf{S}_{t}^{*} f$, we get an identity which expresses the fact that the system $\Sigma$ is time-invariant:

$$
\mathbf{S}_{t}^{*}\left(\Psi_{\infty} z+\mathbb{F}_{\infty} f\right)=\Psi_{\infty}\left(\mathbb{T}_{t} z+\Phi_{t} f\right)+\mathbb{F}_{\infty} \mathbf{S}_{t}^{*} f
$$

Now we consider the state trajectory $x$. We put $x_{0}=x(0)$, then $x$ is given by $(2.15 \mathrm{a})$. Let $y$ be defined by (2.15b) (the output function), so by Proposition 4.1 and by (4.2) we have $y \in L_{\omega}^{2}$. Taking in the last identity $z=x_{0}$ and $f=u$, we get that for any $t \geq 0$

$$
\mathbf{S}_{t}^{*} y=\Psi_{\infty} x(t)+\mathbb{F}_{\infty} \mathbf{S}_{t}^{*} u .
$$

We consider that $u(t)$ is well defined for every $t \geq 0$, i.e., we make a choice from the equivalence class of functions equal a.e. which represent $u$. Let for any $t \geq 0$ the function $\varepsilon_{t} \in \widetilde{\Omega}$ be defined by

$$
\varepsilon_{t}(\sigma)=u(t+\sigma)-u(t),
$$

so $\mathbf{S}_{t}^{*} u=\chi \cdot u(t)+\varepsilon_{t}$. Substituting this into (4.6) and then integrating on $[0, \tau]$ we get, using (2.11)

$$
\begin{aligned}
\int_{0}^{\tau} y(t+\sigma) d \sigma= & C \int_{0}^{\tau} \mathbb{T}_{\sigma} x(t) d \sigma+\int_{0}^{\tau}\left(\mathbb{F}_{\infty}(\chi \cdot u(t))\right)(\sigma) d \sigma \\
& +\int_{0}^{\tau}\left(\mathbb{F}_{\infty} \varepsilon_{t}\right)(\sigma) d \sigma .
\end{aligned}
$$

Dividing everything by $\tau$ and using (4.3) and (4.4) we get

$$
\frac{1}{\tau} \int_{0}^{\tau} y(t+\sigma) d \sigma=C_{L}^{\tau} x(t)+D^{\tau} u(t)+\frac{1}{\tau} \int_{0}^{\tau}\left(\mathbb{F}_{\infty} \varepsilon_{t}\right)(\sigma) d \sigma .
$$

We mention that this formula is needed also in the proof of the representation theorem (Theorem 2.3), as given in the Appendix.

It is easy to see that in the space $L_{\omega}^{2}$ we have $\lim _{\tau \rightarrow 0}(1 / \tau) \int_{0}^{\tau} y(t+\sigma) d \sigma=$ $y(t)$ (both sides are defined for a.e. $t \geq 0$ ). Using the representation of $y$ given in Theorem 2.3, this becomes

$$
\lim _{\tau \rightarrow 0} \frac{1}{\tau} \int_{0}^{\tau} y(t+\sigma) d \sigma=C_{L} x(t)+D u(t) \text { in } L_{\omega}^{2}
$$

(both sides have to be regarded as functions of $t$, defined for a.e. $t \geq 0$ ). If we can prove that

$$
\lim _{\tau \rightarrow 0} \frac{1}{\tau} \int_{0}^{\tau}\left(\mathbb{F}_{\infty} \varepsilon_{t}\right)(\sigma) d \sigma=0 \quad \text { in } L_{\omega}^{2}
$$

(again, both sides are functions of $t$ ), then (4.5), (4.8), (4.9), and (4.10) imply the conclusion of the lemma. So it remains to prove (4.10).

We have for $\tau \in(0,1]$, using that $\mathbf{P}_{\tau} \mathbb{F}_{\infty}=\mathbb{F}_{\tau}$ and $\left\|\mathbb{F}_{\tau}\right\| \leq\left\|\mathbb{F}_{1}\right\|$,

$$
\begin{aligned}
\left\|\frac{1}{\tau} \int_{0}^{\tau}\left(\mathbb{F}_{\infty} \varepsilon_{t}\right)(\sigma) d \sigma\right\| & \leq \frac{1}{\tau} \int_{0}^{\tau}\left\|\left(\mathbb{F}_{\tau} \varepsilon_{t}\right)(\sigma)\right\| d \sigma \leq\left(\frac{1}{\tau} \int_{0}^{\tau}\left\|\left(\mathbb{F}_{\tau} \varepsilon_{t}\right)(\sigma)\right\|^{2} d \sigma\right)^{1 / 2} \\
& \leq\left\|\mathbb{F}_{1}\right\|\left(\frac{1}{\tau} \int_{0}^{\tau}\left\|\varepsilon_{t}(\sigma)\right\|^{2} d \sigma\right)^{1 / 2} .
\end{aligned}
$$


Both sides above are in $L_{\omega}^{2}$, when regarded as functions of $t \in[0, \infty)$. Using Fubini's theorem we get

$$
\begin{aligned}
\left\|\frac{1}{\tau} \int_{0}^{\tau}\left(\mathbb{F}_{\infty} \varepsilon_{t}\right)(\sigma) d \sigma\right\|_{L_{\omega}^{2}}^{2} & \leq\left\|\mathbb{F}_{1}\right\|^{2} \int_{0}^{\infty} e^{-2 \omega t}\left(\frac{1}{\tau} \int_{0}^{\tau}\left\|\varepsilon_{t}(\sigma)\right\|^{2} d \sigma\right) d t \\
& =\left\|\mathbb{F}_{1}\right\|^{2} \frac{1}{\tau} \int_{0}^{\tau} \int_{0}^{\infty} e^{-2 \omega t}\left\|\varepsilon_{t}(\sigma)\right\|^{2} d t d \sigma \\
& =\left\|\mathbb{F}_{1}\right\|^{2} \frac{1}{\tau} \int_{0}^{\tau}\left\|\mathbf{S}_{\sigma}^{*} u-u\right\|_{L_{\omega}^{2}}^{2} d \sigma .
\end{aligned}
$$

Since $\left(\mathbf{S}_{\sigma}^{*}\right)_{\sigma \geq 0}$ is a strongly continuous semigroup on $L_{\omega}^{2}$, it follows that the last expression on the right-hand side above tends to zero as $\tau \rightarrow 0$, i.e., we have proved (4.10).

Remark 4.5. Let $x$ be a state trajectory of $\Sigma$ corresponding to an input $u \in \widetilde{\Omega}$ and let $C_{L}^{\tau}$ be defined as in (4.3).Then

$$
\lim _{\tau \rightarrow 0} C_{L}^{\tau} x=C_{L} x \text { in } \widetilde{\Gamma} \text {. }
$$

To prove this, we have to show that $\mathbf{P}_{T} C_{L}^{\tau} x \rightarrow \mathbf{P}_{T} C_{L} x$ in $L^{2}$, for any $T \geq 0$. So let $T \geq 0$ and let $x_{T}$ be the state trajectory starting from $x(0)$, corresponding to the input $\mathbf{P}_{T} u$. By causality (see the beginning of $\S 3$ ) we have $\mathbf{P}_{T} C_{L}^{\tau} x=\mathbf{P}_{T} C_{L}^{\tau} x_{T}$, and similarly for $C_{L}$ in place of $C_{L}^{\tau}$. Let $\omega>\omega_{0}$. Since $\mathbf{P}_{T} u \in L_{\omega}^{2}$, Lemma 4.4 implies that $\mathbf{P}_{T} C_{L}^{\tau} x \rightarrow \mathbf{P}_{T} C_{L} x$ in $L_{\omega}^{2}$ as $\tau \rightarrow 0$, which is equivalent to convergence in $L^{2}$.

Theorem 4.6. Let $\omega>\omega_{0}$ and $u \in L_{\omega}^{2}([0, \infty), U)$. Let $x$ be a state trajectory of $\Sigma$ corresponding to the input $u$, and let $\alpha \in L_{-\omega}^{2}[0, \infty)$. Then

$$
\int_{0}^{\infty} C_{L} \alpha(t) x(t) d t=C_{L} \int_{0}^{\infty} \alpha(t) x(t) d t
$$

(in particular, $\int_{0}^{\infty} \alpha(t) x(t) d t \in D\left(C_{L}\right)$ ).

Proof. The operators $C_{L}^{\tau}(\tau>0)$ were defined in (4.3). It is easy to verify that the functions $x, C_{L}^{\tau} x$ and $C_{L} x$ are in $L_{\omega}^{2}$ (use Proposition 3.2 for $x$ and $C_{L}^{\tau} x$ and Proposition 4.1 and (4.2) for $C_{L} x$ ). It follows that the functions $\alpha x$, $C_{L}^{\tau} \alpha x$ and $C_{L} \alpha x$ are in $L^{1}$ and, by Lemma 4.4,

$$
\lim _{\tau \rightarrow 0} C_{L}^{\tau} \alpha x=C_{L} \alpha x \text { in } L^{1}([0, \infty), Y) \text {. }
$$

Since $C_{L}^{\tau}$ commutes with the integral sign, we get

$$
\int_{0}^{\infty} C_{L} \alpha(t) x(t) d t=\lim _{\tau \rightarrow 0} C_{L}^{\tau} \int_{0}^{\infty} \alpha(t) x(t) d t .
$$

By the definition of $C_{L}$ (see (2.12)), this implies that $\int_{0}^{\infty} \alpha(t) x(t) d t \in D\left(C_{L}\right)$ and (4.11) holds.

We mention that a weaker version of Theorem 4.6, valid for PritchardSalamon systems and finite dimensional $U$, was given in $[5, \S 3]$.

We restate Theorem 1.1 in a somewhat more precise way. Remember that we are still using the notation introduced before Lemma 4.4. Now, all Hilbert spaces are assumed to be complex. 
Theorem 4.7. For $s \in \mathbb{C}_{\omega_{0}}$, the transfer function $\mathbf{H}$ of $\Sigma$ is given by (1.1), i.e.,

$$
\mathbf{H}(s)=C_{L}(s I-A)^{-1} B+D,
$$

in particular, $(s I-A)^{-1} B U \subset D\left(C_{L}\right)$.

Proof. Let $\omega>\omega_{0}$ and $u \in L_{\omega}^{2}$. Let $x$ and $y$ be defined by (2.15), with $x_{0}=0$, so by Theorem 2.3 they satisfy equations (1.2) for a.e. $t \geq 0$. By Proposition 3.2 applied to the operator $u \rightarrow x$, we have $x \in L_{\omega}^{2}$, and by Proposition 4.1 we have $y \in L_{\omega}^{2}$. We denote by $\hat{u}, \hat{x}$, and $\hat{y}$ the Laplace transforms of $u, x$, and $y$. Equation (1.2a) implies

$$
\hat{x}(s)=(s I-A)^{-1} B \hat{u}(s), \quad \forall s \in \mathbb{C}_{\omega} .
$$

Applying the Laplace transformation to $(1.2 \mathrm{~b})$, we get that for $s \in \mathbb{C}_{\omega}$

$$
\hat{y}(s)=\int_{0}^{\infty} e^{-s t} C_{L} x(t) d t+D \hat{u}(s) .
$$

According to Theorem 4.6 (with $\alpha(t)=e^{-s t}$ ) we can take $C_{L}$ out of the integral, getting $\hat{x}(s) \in D\left(C_{L}\right)$ and

$$
\hat{y}(x)=C_{L} \hat{x}(s)+D \hat{u}(s), \quad \forall s \in \mathbb{C}_{\omega} .
$$

Combined with (4.12) this proves (1.1) for $s \in \mathbb{C}_{\omega}$. The argument being valid for any $\omega>\omega_{0}$, it follows that (1.1) holds on $\mathbb{C}_{\omega_{0}}$.

Remark 4.8. The transfer function $\mathbf{H}$ is defined on $\mathbb{C}_{\gamma_{0}}$, which in general is larger than $\mathbb{C}_{\omega_{0}}$ (see Proposition 4.1). In the strip $\gamma_{0}<\operatorname{Re} s \leq \omega_{0}$ formula (1.1) in general no longer holds, not even for $s \in \rho(A)$. This is illustrated by the following example, which was shown to me by Hans Zwart [20].

Consider $X=l^{2}(\mathbb{Z}), U=Y=\mathbb{C}$, and let $\left(g_{k}\right)_{k \in \mathbb{Z}}$ denote the standard basis of $X$. Let $A$ be the right shift on $X$ (i.e., $A g_{k}=g_{k+1}$ ), let $B=g_{1}$, $C=g_{0}$ (i.e., $C z=\left\langle z, g_{0}\right\rangle$ ), and $D=0$. Let $\Sigma$ be the system determined by $(A, B, C, D)$ via (1.2). Since $C A^{k} B=0$ for any $k \in\{0,1,2, \ldots\}$, it follows that $C e^{A t} B=0$ for any $t \geq 0$, whence $\gamma_{0}=-\infty$ and $\mathbf{H}=0$. The spectrum $\sigma(A)$ is the unit circle, whence $\omega_{0}=1$. If we evaluate $C(s I-A)^{-1} B$ for $s=0$, we get -1 , but $\mathbf{H}(0)=0$.

Remark 4.9. It follows from (1.1) and the resolvent identity that for any $s, \beta \in$ $\mathbb{C}_{\omega_{0}}$ with $s \neq \beta$

$$
\frac{\mathbf{H}(s)-\mathbf{H}(\beta)}{s-\beta}=-C(s I-A)^{-1}(\beta I-A)^{-1} B .
$$

In the above formula we do not need $C_{L}$ (instead of $C$ ) because

$$
(s I-A)^{-1}(\beta I-A)^{-1} B U \subset X_{1} .
$$

If $A, B$ and $C$ are given and we want to compute $\mathbf{H}$, then (4.13) allows us to compute $\mathbf{H}$ on $\mathbb{C}_{\omega_{0}}$ up to an additive constant operator, without having to compute $C_{L}$ first (as in (1.1)).

Remark 4.10. Formula (4.13) remains valid for any abstract linear system (without having to assume regularity), see Salamon [15] for a direct proof. It is interesting to note that (4.13) (for abstract linear systems) can be obtained as a consequence of (1.1) (valid for regular linear systems). 
We sketch the proof. Let $\Sigma$ be an abstract linear system and let $\Sigma^{c}$ be the system obtained by the cascade connection of $\Sigma$ followed by an integrator. Clearly $\Sigma^{c}$ has continuous step responses, so that it is regular. If $\mathbf{H}$ and $\mathbf{H}^{c}$ are the transfer functions of $\Sigma$ and $\Sigma^{c}$, then $\mathbf{H}^{c}(s)=(1 / s) \mathbf{H}(s)$. The state space of $\Sigma^{c}$ is $X \times Y$, where $X$ and $Y$ are the state and output spaces of $\Sigma$. If $A$ and $C$ are the semigroup generator and observation operator of $\Sigma$, then the corresponding operators for $\Sigma^{c}$ are

$$
A^{c}=\left(\begin{array}{ll}
A & 0 \\
C & 0
\end{array}\right), \quad C^{c}=\left(\begin{array}{ll}
0 & I
\end{array}\right),
$$

with $D\left(A^{c}\right)=D(A) \times Y$. Let $B$ and $B^{c}$ denote the control operators of $\Sigma$ and $\Sigma^{c}$ (it seems that there is no simple way to express $B^{c}$ in terms of $A, B, C$ ). Then by (1.1), $\mathbf{H}^{c}(s)=C^{c}\left(s I-A^{c}\right)^{-1} B^{c}$. We can get $B$ and $B^{c}$ as strong limits, for $\tau \rightarrow 0$, of $B^{\tau}$ and $B^{c \tau}$, which are families of bounded control operators, see (2.6). Moreover, the first component of $B^{c \tau}$ is exactly $B^{\tau}$. If we denote $\mathbf{H}^{c \tau}(s)=C^{c}\left(s I-A^{c}\right)^{-1} B^{c \tau}$, then (by an elementary computation) $s \mathbf{H}^{c \tau}(s)-\beta \mathbf{H}^{c \tau}(\beta)=C\left[(s I-A)^{-1}-(\beta I-A)^{-1}\right] B^{\tau}$. Applying both sides to any fixed element of the input space, taking limits as $\tau \rightarrow 0$ and using the resolvent identity, we get (4.13).

Remark 4.11. Abstract boundary control systems, as introduced in Salamon [14, $\S 2.2]$, are abstract linear systems with $B$ injective and such that $X \cap B U=\{0\}$. Salamon proved that such systems have a relatively simple representation in terms of a differential and two algebraic equations, valid for smooth inputs and compatible initial conditions (see [14]). Part of his construction is the introduction of the Hilbert space $Z=\{z \in X \mid A z \in X+B U\}$ and of the operators $\Gamma \in \mathscr{L}(Z, U)$ and $K \in \mathscr{L}(Z, Y)$ such that $(A+B \Gamma) z \in X$ for any $z \in Z$ (this determines $\Gamma$ ) and

$$
\mathbf{H}(s)=K(s I-A)^{-1} B, \quad \forall s \in \mathbb{C}_{\omega_{0}} .
$$

It is somewhat confusing that both $C_{L}$ and $K$ are extensions of $C$ and appear in formulae expressing $\mathbf{H}$, but otherwise have little in common. However, for regular boundary control systems we have $Z \subset D\left(C_{L}\right)$ and the relationship between the restriction of $C_{L}$ to $Z$ and $K$ is simple:

$$
K z=\left(C_{L}+D \Gamma\right) z, \quad \forall z \in Z
$$

(this follows by a short computation).

Remark 4.12. Everything in this section remains valid, with minor and obvious modifications, if we replace Hilbert spaces by Banach spaces and $L^{2}$ by $L^{p}$, with $1 \leq p<\infty$ (in Theorem 4.6 we have to assume $\alpha \in L_{-\omega}^{q}[0, \infty$ ), where $1 / p+1 / q=1)$. For $p=\infty$, only Proposition 4.1 remains valid.

\section{A TAUBERIAN THEOREM ABOUT THE LAPLACE TRANSFORMATION ON $L^{2}$, AND THE SPECTRAL CHARACTERIZATION OF REGULARITY}

First a few words on terminology, following Doetsch [8]. Let $y$ be a function on $[0, \infty)$, which has a Laplace transform $\hat{y}$. A theorem which tells something about the asymptotic behavior of $\hat{y}$, based on the asymptotic behavior of $y$, is called an Abelian theorem. Usually, the converse of an Abelian theorem is not true, unless we impose an additional condition on $y$, called a Tauberian 
condition. Such a partial converse is called a Tauberian theorem. There is lately a renewed interest in Tauberian theory, see, for example, Arendt and Prüss [1], Batty [2], and the references therein.

Now we are interested in functions that belong to $L^{2}([0, \infty), Y)$, where $Y$ is a Hilbert space (for Banach spaces see Remark 5.9). First we state a simple Abelian theorem (see Doetsch [8, p. 473] for the scalar version).

Proposition 5.1. Let $y \in L^{2}([0, \infty), Y)$ be such that for $t \in(0, \infty)$

$$
\lim _{t \rightarrow 0} \frac{1}{t} \int_{0}^{t} y(\sigma) d \sigma=d
$$

Then for $\lambda \in(0, \infty)$

$$
\lim _{\lambda \rightarrow \infty} \lambda \hat{y}(\lambda)=d
$$

Proof. For any $t>0$

$$
\int_{0}^{t} y(\sigma) d \sigma=t d+t \varepsilon(t)
$$

where $\varepsilon$ is a continuous and bounded $Y$-valued function on $(0, \infty)$, with $\|\varepsilon(t)\| \rightarrow 0$ for $t \rightarrow 0$. Applying the Laplace transformation, we get for $\lambda>0$

$$
\frac{1}{\lambda} \hat{y}(\lambda)=\frac{1}{\lambda^{2}} d+\int_{0}^{\infty} e^{-\lambda t} t \varepsilon(t) d t
$$

whence

$$
\|\lambda \hat{y}(\lambda)-d\| \leq \lambda^{2} \int_{0}^{\infty} e^{-\lambda t} t\|\varepsilon(t)\| d t .
$$

It is an easy exercise to show that the right-hand side above tends to zero as $\lambda \rightarrow \infty$. (Hint: decompose like $\int_{0}^{\infty}=\int_{0}^{\tau}+\int_{\tau}^{\infty}$, with $\tau=1 / \sqrt{\lambda}$.)

The following partial converse of Proposition 5.1 is known (see Doetsch [8, p. 512]): if $Y=\mathbb{R}$ and the Tauberian condition $\limsup _{t \rightarrow 0} y(t)<\infty$ (or $\left.\liminf _{t \rightarrow 0} y(t)>-\infty\right)$ holds, then (5.2) implies (5.1).

We shall prove another partial converse of Proposition 5.1 (our Tauberian condition is (5.3)).

Theorem 5.2. Suppose $y \in L^{2}([0, \infty), Y)$ is such that

$$
\sup _{t>0} \frac{1}{t} \int_{0}^{t}\|y(\sigma)\|^{2} d \sigma<\infty
$$

If the Laplace transform $\hat{y}$ satisfies (5.2), then $y$ satisfies (5.1).

Proof. Let us denote the left-hand side of (5.3) by $M^{2}$. First we prove that

$$
\lambda \int_{0}^{\infty} e^{-\lambda t}\|y(t)\| d t \leq M, \quad \forall \lambda>0 .
$$

Indeed, we have for any $\lambda>0$

$$
\begin{aligned}
\lambda \int_{0}^{\infty} e^{-\lambda t}\|y(t)\| d t & =\lambda^{2} \int_{0}^{\infty} e^{-\lambda t}\left(\int_{0}^{t}\|y(\sigma)\| d \sigma\right) d t \\
& \leq \lambda^{2} \int_{0}^{\infty} e^{-\lambda t} t\left(\frac{1}{t} \int_{0}^{t}\|y(\sigma)\|^{2} d \sigma\right)^{1 / 2} d t \\
& \leq M \lambda^{2} \int_{0}^{\infty} e^{-\lambda t} t d t=M .
\end{aligned}
$$



have

The next step is to show that for any continuous function $f:[0,1] \rightarrow \mathbb{R}$ we

$$
\lim _{\lambda \rightarrow \infty} \lambda \int_{0}^{\infty} e^{-\lambda t} f\left(e^{-\lambda t}\right) y(t) d t=d \int_{0}^{\infty} e^{-t} f\left(e^{-t}\right) d t
$$

We have

$$
\begin{aligned}
\lim _{\lambda \rightarrow \infty} \lambda \int_{0}^{\infty} e^{-\lambda t}\left(e^{-\lambda t}\right)^{n} y(t) d t & =\frac{1}{n+1} \lim _{\lambda \rightarrow \infty} \lambda(n+1) \hat{y}(\lambda(n+1)) \\
& =\frac{1}{n+1} d=d \int_{0}^{\infty} e^{-t}\left(e^{-t}\right)^{n} d t
\end{aligned}
$$

which implies that (5.5) is true for any polynomial $f$. We denote for any bounded, measurable $g:[0,1] \rightarrow \mathbb{R}$ and any $\lambda>0$

$$
E(\lambda, g)=\lambda \int_{0}^{\infty} e^{-\lambda t} g\left(e^{-\lambda t}\right) y(t) d t
$$

Let $f:[0,1] \rightarrow \mathbb{R}$ be continuous and let $\left(f_{n}\right)$ be a sequence of polynomials such that $f_{n} \rightarrow f$ uniformly. Then (5.4) implies

$$
\begin{aligned}
\left\|E\left(\lambda, f_{n}\right)-E(\lambda, f)\right\| & \leq \lambda \int_{0}^{\infty} e^{-\lambda t}\left|f_{n}\left(e^{-\lambda t}\right)-f\left(e^{-\lambda t}\right)\right| \cdot\|y(t)\| d t \\
& \leq M \sup _{x \in[0,1]}\left|f_{n}(x)-f(x)\right|,
\end{aligned}
$$

so $E\left(\lambda, f_{n}\right) \rightarrow E(\lambda, f)$ uniformly with respect to $\lambda$. Therefore

$$
\lim _{\lambda \rightarrow \infty} E(\lambda, f)=\lim _{n \rightarrow \infty} \lim _{\lambda \rightarrow \infty} E\left(\lambda, f_{n}\right),
$$

which is the same as (5.5).

The next step is to prove (5.5) for $f$ replaced by a particular discontinuous function $f_{0}:[0,1] \rightarrow \mathbb{R}$, defined by

$$
f_{0}(x)= \begin{cases}0, & \text { for } x \in\left[0, e^{-1}\right) \\ 1 / x, & \text { for } x \in\left[e^{-1}, 1\right]\end{cases}
$$

We approximate $f_{0}$ by a family of continuous functions $f_{\varepsilon}, \varepsilon>0$, defined as follows: $f_{\varepsilon}(x)=f_{0}(x)$ for any $x$ in $[0,1]$ except in the subinterval $\left[e^{-1-\varepsilon}, e^{-1}\right)$, and $f_{\varepsilon}$ interpolates linearly inside this subinterval, i.e.,

$$
f_{\varepsilon}(x)=\frac{x-e^{-1-\varepsilon}}{e^{-1}-e^{-1-\varepsilon}} \cdot e, \quad \text { for } x \in\left[e^{-1-\varepsilon}, e^{-1}\right) .
$$

It is elementary to check that for any $t, \lambda>0$

$$
e^{-\lambda t}\left|f_{\varepsilon}\left(e^{-\lambda t}\right)-f_{0}\left(e^{-\lambda t}\right)\right| \leq \chi_{[1 / \lambda, 1 / \lambda(1+\varepsilon)]}(t)
$$

where $\chi_{J}$ denotes the indicator function of the interval $J$. We have, using the above inequality,

$$
\begin{aligned}
\left\|E\left(\lambda, f_{\varepsilon}\right)-E\left(\lambda, f_{0}\right)\right\| & \leq \lambda \int_{1 / \lambda}^{(1+\varepsilon) / \lambda}\|y(t)\| d t \\
& \leq \sqrt{\varepsilon}\left(\lambda \int_{1 / \lambda}^{(1+\varepsilon) / \lambda}\|y(t)\|^{2} d t\right)^{1 / 2}
\end{aligned}
$$


Assuming for convenience $\varepsilon \leq 1$ and denoting $2 / \lambda=\tau$, we get by (5.3)

$$
\left\|E\left(\lambda, f_{\varepsilon}\right)-E\left(\lambda, f_{0}\right)\right\| \leq \sqrt{2 \varepsilon}\left(\frac{1}{\tau} \int_{0}^{\tau}\|y(t)\|^{2} d t\right)^{1 / 2} \leq \sqrt{2 \varepsilon} \cdot M,
$$

which shows that $E\left(\lambda, f_{\varepsilon}\right) \rightarrow E\left(\lambda, f_{0}\right)$ uniformly with respect to $\lambda$. Therefore

$$
\lim _{\lambda \rightarrow \infty} E\left(\lambda, f_{0}\right)=\lim _{\varepsilon \rightarrow 0} \lim _{\lambda \rightarrow \infty} E\left(\lambda, f_{\varepsilon}\right) \text {. }
$$

Since (5.5) holds for $f_{\varepsilon}$ in place of $f$, we have now obtained that it also holds for $f_{0}$ in place of $f$, i.e.,

$$
\lim _{\lambda \rightarrow \infty} \lambda \int_{0}^{1 / \lambda} y(t) d t=d \int_{0}^{1} d t
$$

which is exactly (5.1).

Problem 5.3. I was not able to construct a function $y \in L^{2}[0, \infty)$ satisfying (5.2) but not (5.1). Such a function would show that the Tauberian condition (5.3) cannot be omitted in Theorem 5.2. For any $p \in[1,2)$, we can construct such a counterexample in $L^{p}[0, \infty)$, as follows:

Let $f:[0, \infty) \rightarrow \mathbb{R}$ be defined by

$$
f(t)= \begin{cases}\sin 2 \pi t & \text { for } t \in[0,1] \\ 0 & \text { for } t>1\end{cases}
$$

and put

$$
y(t)=\sum_{k=1}^{\infty} k^{2 / p} g_{k} f(k(k+1) t-k),
$$

where

$$
g_{k}= \begin{cases}1 / n^{2} & \text { for } k=2^{n}, n \in \mathbb{N} \\ 0 & \text { for all other } k \in \mathbb{N}\end{cases}
$$

By some standard computations which we do not display here, $y \in L^{p}[0, \infty)$, (5.2) holds with $d=0$, but the limit in (5.1) does not exist.

It would be a very interesting result, if it could be proved, that for $p \geq 2$ we do not need (5.3) in Theorem 5.2 (see also Remarks 5.7 and 5.9).

The following proposition is a strengthening of Proposition 5.1. Recall the notation $\alpha_{2}(y)$, defined in $\S 3$. We introduce a notation for angular domains in $\mathbb{C}:$ for any $\psi \in(0, \pi]$

$$
\mathscr{W}(\psi)=\left\{r e^{i \phi} \mid r \in(0, \infty), \phi \in(-\psi, \psi)\right\} .
$$

Proposition 5.4. Suppose $y \in L_{\text {loc }}^{2}([0, \infty), Y)$ is such that $\alpha_{2}(y)<\infty$ and $(5.1)$ holds. Then for any $\psi \in(0, \pi / 2)$

$$
\lim _{s \in \mathscr{W}(\psi),|s| \rightarrow \infty} s \hat{y}(s)=d .
$$

Proof. This can be obtained by slightly modifying the proof of Proposition 5.1. Now the function $\varepsilon$ will not be bounded in general, but it can be written in the form $\varepsilon(t)=\varepsilon_{0}(t) e^{\alpha t}$, where $\varepsilon_{0}$ is bounded and $\alpha>\alpha_{2}(y)$. The variable $\lambda>0$ has to be replaced with $s$ (and sometimes with $|s|$ ), where $s \in \mathscr{W}(\psi)$ and $\operatorname{Re} s>\alpha$. 
We cannot allow $\psi=\pi / 2$ in (5.8), as the simple example $\hat{y}(s)=e^{-s} /(s+1)$ shows. We did not state Proposition 5.1 in this strengthened form from the beginning, because this would have obscured its relationship to Theorem 5.2. The following corollary is a strengthening of Theorem 5.2.

Corollary 5.5. Suppose $y \in L_{\mathrm{loc}}^{2}([0, \infty), Y)$ is such that $\alpha_{2}(y)<\infty$ and

$$
\limsup _{t \rightarrow 0} \frac{1}{t} \int_{0}^{t}\|y(\sigma)\|^{2} d \sigma<\infty .
$$

If the Laplace transform $\hat{y}$ satisfies (5.2), then $y$ satisfies (5.1).

Proof. Decompose $y=y_{1}+y_{2}$, where $y_{1}$ is supported on $[0,1]$ and $y_{2}$ on $[1, \infty)$. By Proposition 5.4, $\lim _{\lambda \rightarrow \infty} \lambda \hat{y}_{2}(\lambda)=0$. It follows that $\lim _{\lambda \rightarrow \infty} \lambda \hat{y}_{1}(\lambda)$ $=d$, and (5.9) implies that $y_{1}$ also satisfies (5.3). By Theorem 5.2 we get that $y_{1}$ satisfies (5.1), i.e., $y$ satisfies (5.1).

We return now to systems theory.

Definition 5.6. Let $\Sigma$ be an abstract linear system, with state space $X$, semigroup generator $A$ and observation operator $C$. We define the operator $\widetilde{C}_{L}$ by

$$
\widetilde{C}_{L} x_{0}=\lim _{\lambda \rightarrow+\infty} C \lambda(\lambda I-A)^{-1} x_{0},
$$

where $\lambda \in \mathbb{R}$, with domain

$$
D\left(\widetilde{C}_{L}\right)=\left\{x_{0} \in X \mid \text { the limit in (5.10) exists }\right\} .
$$

Remark 5.7. Denoting by $C_{L}$ the Lebesgue extension of $C$ (see (2.12)), we have that $\widetilde{C}_{L}$ is an extension of $C_{L}$, i.e.,

$$
\begin{gathered}
D\left(C_{L}\right) \subset D\left(\widetilde{C}_{L}\right) \subset X, \\
\widetilde{C}_{L} x=C_{L} x, \quad \forall x \in D\left(C_{L}\right) .
\end{gathered}
$$

This follows from Proposition 5.4 with $y(t)=\Psi_{\infty} x_{0}$, where $\Psi_{\infty}$ is the extended output map of $\Sigma$ (see $\S 2$ ), using (2.11) and (4.2). (A direct proof was given in Weiss [17, Proposition 4.7], but without defining $\widetilde{C}_{L}$.)

I do not know if the first inclusion in (5.11) can be strict. In order to answer this question, a solution of Problem 5.3 would be needed. In any case, we will see below that for any practical purpose $C_{L}$ and $\widetilde{C}_{L}$ are interchangeable, we can always use the one which suits us better.

The following theorem contains Theorem 1.3.

Theorem 5.8. Let $\Sigma=(\mathbb{T}, \Phi, \Psi, \mathbb{F})$ be an abstract linear system, with input space $U$, state space $X$, output space $Y$, semigroup generator $A$, control operator $B$, observation operator $C$, and transfer function $\mathbf{H}$. The operators $C_{L}$ and $\widetilde{C}_{L}$ are defined in (2.12) and (5.10), and $\omega_{0}$ is the growth bound of $\mathbb{T}$. Then the following statements are equivalent.

(1) $\Sigma$ is regular, i.e., for any $\mathrm{v} \in U$ the limit in (2.14) exists.

(2) For any $s \in \rho(A)$ we have that $(s I-A)^{-1} B U \subset D\left(C_{L}\right)$ and $C_{L}(s I-A)^{-1} B$ is an analytic $\mathscr{L}(U, Y)$-valued function of $s$ on $\rho(A)$, bounded on any half-plane $\mathbb{C}_{\omega}$ with $\omega>\omega_{0}$. 
(3) There exists $s \in \rho(A)$ such that $(s I-A)^{-1} B U \subset D\left(C_{L}\right)$.

(4) There exists $s \in \rho(A)$ such that $(s I-A)^{-1} B U \subset D\left(\widetilde{C}_{L}\right)$.

(5) Any state trajectory of $\Sigma$ is a.e. in $D\left(C_{L}\right)$.

(6) Any state trajectory of $\Sigma$ is a.e. in $D\left(\widetilde{C}_{L}\right)$.

(7) For any $\mathrm{v} \in U$ and any $\psi \in(0, \pi / 2), \mathbf{H}(s) \mathbf{v}$ has a limit as $|s| \rightarrow \infty$ in $\mathscr{W}(\psi)($ see $(5.7))$.

(8) For any $\mathbf{v} \in U, \mathbf{H}(\lambda) \mathbf{v}$ has a limit as $\lambda \rightarrow+\infty$ in $\mathbb{R}$.

Moreover, if the limits mentioned in (1), (7), and (8) above exist, then they are equal to $D \mathrm{v}$, where $D$ is the feedthrough operator of $\Sigma$.

Proof. Our plan is to prove the implications in the following diagram

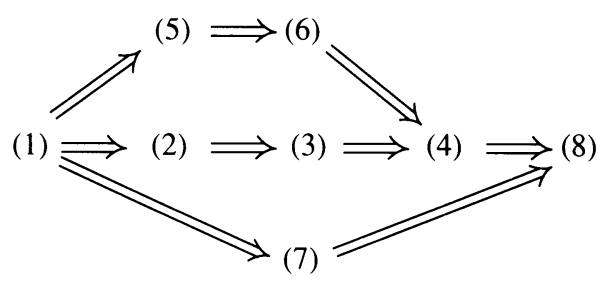

This will show that (1) implies any other point and any other point implies (8). Then, we will close the loop by proving that (8) implies (1) (it is here that we need the Tauberian theorem proved earlier).

The implication $(1) \Rightarrow(2)$ : Suppose (1) holds. Theorem 4.7 implies that for $s \in \mathbb{C}_{\omega_{0}}$ we have $(s I-A)^{-1} B U \subset D\left(C_{L}\right)$ and $C_{L}(s I-A)^{-1} B \in \mathscr{L}(U, Y)$. By the resolvent identity we obtain that these properties remain true for any $s \in \rho(A)$ and the function $\mathbf{G}$ defined on $\rho(A)$ by $\mathbf{G}(s)=C_{L}(s I-A)^{-1} B$ satisfies (4.13) for any $s, \beta \in \rho(A)$ with $s \neq \beta$. It is easy to show (using again the resolvent identity) that $C(s I-A)^{-1}$ is an analytic $\mathscr{L}(X, Y)$-valued function of $s$ on $\rho(A)$. Now (4.13) (with $\mathbf{G}$ in place of $\mathbf{H}$ ) implies that also $\mathbf{G}$ is analytic on $\rho(A)$. It remains to show the boundedness of $\mathbf{G}$ on certain half-planes. By Theorem 4.7 we have $\mathbf{G}(s)=\mathbf{H}(s)-D$ for $s \in \mathbb{C}_{\omega_{0}}$. By Theorem 3.6 and Proposition 4.1, $\mathbf{H}$ is bounded on $\mathbb{C}_{\omega}$ for any $\omega>\omega_{0}$, so the same applies to $\mathbf{G}$.

The implication $(2) \Rightarrow(3)$ is trivial.

The implication $(3) \Rightarrow(4)$ follows from (5.11).

The implication $(4) \Rightarrow(8)$ : Suppose (4) holds. By the resolvent identity we get that $(s I-A)^{-1} B U \subset D\left(\widetilde{C}_{L}\right)$ for any $s \in \rho(A)$. We denote, for any $s \in \rho(A), \widetilde{\mathbf{G}}(s)=\widetilde{C}_{L}(s I-A)^{-1} B$, which by (5.10) means that for any $\mathrm{v} \in U$

$$
\lim _{\lambda \rightarrow \infty} C \lambda(\lambda I-A)^{-1}(s I-A)^{-1} B \mathbf{v}=\widetilde{\mathbf{G}}(s) \mathbf{v} .
$$

Since for any $z \in X, \lim _{\lambda \rightarrow \infty}(\lambda I-A)^{-1} z=0$ in $X_{1}$ and $C$ is bounded on $X_{1}$, it follows that for any $s \in \rho(A)$ and any $\mathrm{v} \in U$

$$
\lim _{\lambda \rightarrow \infty} C s(\lambda I-A)^{-1}(s I-A)^{-1} B \mathrm{v}=0 .
$$

Subtracting this side by side from (5.12) and using the resolvent identity, we get that $\lim _{\lambda \rightarrow \infty}[\widetilde{\mathbf{G}}(s)-\widetilde{\mathbf{G}}(\lambda)] \mathbf{v}=\widetilde{\mathbf{G}}(s) \mathbf{v}$, which shows that

$$
\lim _{\lambda \rightarrow \infty} \widetilde{\mathbf{G}}(\lambda) \mathbf{v}=0, \quad \forall \mathbf{v} \in U \text {. }
$$


By the resolvent identity, $\widetilde{\mathbf{G}}(s)$ satisfies (4.13) for any $s, \beta \in \rho(A)$ with $s \neq \beta$. By Remark 4.10, $\mathbf{H}$ satisfies (4.13) for any $s, \beta \in \mathbb{C}_{\omega_{0}}$ with $s \neq \beta$. It follows that for $s \in \mathbb{C}_{\omega_{0}}, \mathbf{H}(s)-\widetilde{\mathbf{G}}(s)$ is independent of $s$. This, together with (5.13) implies (8).

The implication $(1) \Rightarrow(5)$ is contained in Theorem 2.3.

The implication $(5) \Rightarrow(6)$ follows from (5.11).

The implication $(6) \Rightarrow(4)$ : Let $s \in \rho(A)$ and $\mathrm{v} \in U$ and denote $x_{0}=$ $(s I-A)^{-1} B \mathrm{v}$. Then the state trajectory of $\Sigma$ starting from $x_{0}$, corresponding to the input $u(t)=e^{s t} \mathrm{v}$, is given by $x(t)=e^{s t} x_{0}$ (indeed, it is clear that these functions satisfy (1.2a)). If (6) holds then this implies $x_{0} \in D\left(\widetilde{C}_{L}\right)$. Since the choice of $\mathrm{v} \in U$ was arbitrary, by the definition of $x_{0}$, (4) holds.

The implication $(1) \Rightarrow(7)$ : For any $\mathrm{v} \in U$, let $y_{\mathrm{v}}$ be the step response of $\Sigma$ corresponding to $\mathbf{v}$ (see $\S 2$ ). By (ii) of Theorem 3.6, $s \hat{y}_{\mathrm{v}}(s)=\mathbf{H}(s) \mathbf{v}$ holds for $\operatorname{Re} s$ sufficiently big. If (1) holds then by Proposition 5.4 applied to $y_{\mathrm{v}}$ we get that for any $\psi \in(0, \pi / 2)$

$$
\lim _{s \in \mathscr{W}(\psi),|s| \rightarrow \infty} \mathbf{H}(s) \mathbf{v}=D \mathbf{v},
$$

where $D$ is the feedthrough operator of $\Sigma$. In particular, (7) is satisfied.

The implication $(7) \Rightarrow(8)$ is trivial.

The implication $(8) \Rightarrow(1)$ : Let $\chi$ denote the constant function on $[0, \infty)$ equal to 1 everywhere, and for any $\mathrm{v} \in U, y_{\mathrm{v}}=\mathbb{F}_{\infty}(\chi \cdot \mathrm{v})$ is the corresponding step response. The function $y_{\mathrm{v}}$ satisfies (5.9). Indeed, we have for $t \in(0,1]$, using that $\mathbf{P}_{t} \mathbb{F}_{\infty}=\mathbb{F}_{t}$ and $\left\|\mathbb{F}_{t}\right\| \leq\left\|\mathbb{F}_{1}\right\|$,

$$
\frac{1}{t} \int_{0}^{t}\left\|y_{\mathrm{v}}(\sigma)\right\|^{2} d \sigma \leq\left\|\mathbb{F}_{1}\right\|^{2} \frac{1}{t} \int_{0}^{t}\|(\chi \cdot \mathrm{v})(\sigma)\|^{2} d \sigma=\left\|\mathbb{F}_{1}\right\|^{2}\|\mathrm{v}\|^{2} .
$$

If (8) holds then $y_{\mathrm{v}}$ satisfies also (5.2). Indeed, we have seen earlier in this proof that $s \hat{y}_{\mathrm{v}}(s)=\mathbf{H}(s) \mathbf{v}$ holds for $\operatorname{Re} s$ sufficiently big, for any $\mathbf{v} \in U$. By Corollary 5.5 it follows that $y_{\mathrm{v}}$ satisfies $(5.1)$, i.e., $\Sigma$ is regular.

Finally, if the limits mentioned in (1), (7), and (8) exist then, by (2.14) and (5.14), they are equal to $D v$.

The above proof might give the wrong impression that in order to prove the equivalence of any two of the eight statements listed in Theorem 5.8, we need the nontrivial implication $(8) \Rightarrow(1)$. This is not the case, for example, in Weiss [18] the equivalence of (1), (2), and (3) was proved by other methods. (Actually, a slightly weaker version of (2) was stated there.)

Remark 5.9. Let us see what happens if we replace in this section Hilbert spaces by Banach spaces and $L^{2}$ by $L^{p}$, where $1 \leq p \leq \infty$. In particular, for $p<\infty$, condition (5.3) has to be written in the form

$$
\sup _{t>0} \frac{1}{t} \int_{0}^{t}\|y(\sigma)\|^{p} d \sigma<\infty,
$$

and (5.9) has to be modified similarly. Everywhere, $\alpha_{2}(y)$ has to be replaced by $\alpha_{p}(y)$, see Remark 3.8. Problem 5.3 remains unchanged.

The case $1<p<\infty$. Everything remains valid after minor modifications. The expression $\sqrt{\varepsilon}$ appearing towards the end of the proof of Theorem 5.2 becomes $\varepsilon^{1 / q}$, where $1 / p+1 / q=1$. For $p<2$ we know that the first inclusion 
in (5.11) can be strict. Indeed, define $X=L^{p}[0, \infty),\left(\mathbb{T}_{t} z\right)(\xi)=z(\xi+t)$ (the left shift semigroup), and $C z=z(0)$ for $z \in X_{1}$. Then the function $y$ constructed in Problem 5.3 is in $D\left(\widetilde{C}_{L}\right)$, but not in $D\left(C_{L}\right)$.

The case $p=1$. Propositions 5.1 and 5.4 still hold, but no longer does Theorem 5.2. A counterexample can be obtained by a slight modification of the counterexample in Problem 5.3, namely, replacing $1 / n^{2}$ by $1 / k$ in the definition of $g_{k}$. In short, what goes wrong in the proof is that now $q=\infty$, so $\varepsilon^{1 / q}$ does not tend to zero. Obviously, Corollary 5.5 fails as well. But the point is that now we do not need Corollary 5.5 in order to prove the implication $(8) \Rightarrow(1)$ in Theorem 5.8. Indeed, if $p=1$ then (1) (and hence all the other statements in Theorem 5.8) always holds, i.e., any abstract linear system is regular. This will follow from results in Part II. The proof of the other implications in the proof of Theorem 5.8 goes similarly as for $p=2$. If $X$ is reflexive then $B$ is bounded, see Weiss [16, Theorem 4.8].

The case $p=\infty$. Propositions 5.1 and 5.4 hold, with the same proof. Condition (5.3) is meaningless but Theorem 5.2 holds without having to impose any condition instead of (5.3). The proof is actually somewhat simpler than in the case $p=2$, but follows the same idea. Corollary 5.5 holds without having to assume (5.9). Theorem 5.8 is meaningless, because possibly $\Sigma$ has no transfer function (see Remark 3.8). A version of the whole theory can be built in which $L^{\infty}$-functions are replaced by regulated functions (these are uniform limits of step functions, they form a closed subspace of $L^{\infty}$ ). Then $C$ is bounded (see Weiss [17, Proposition 6.5]) and the statements in Theorem 5.8 always hold (like for $p=1$ ). We do not discuss this further.

\section{APPENDIX: THE PROOF OF THEOREM 2.3}

For the proof of Theorem 2.3 we have quoted Weiss [18]. This paper appeared in the proceedings of a conference, and so it might not be available to some readers. The aim of this short section is to give the proof of this theorem, actually in more detail than it was done in [18], where the proof of the following lemma was omitted.

Lemma 6.1. Let $U$ be a Hilbert space and $u \in L_{\mathrm{loc}}^{2}([0, \infty), U)$. Then for almost every $t \geq 0$

$$
\lim _{\tau \rightarrow 0} \frac{1}{\tau} \int_{0}^{\tau}\|u(t+\sigma)-u(t)\|^{2} d \sigma=0 .
$$

Proof. After changing the values of $u$ on a set of measure zero, its range becomes separable, i.e., $u([0, \infty))$ has a countable dense subset $\left\{\mathrm{v}_{n} \mid n \in \mathbb{N}\right\}$. For any $n \in \mathbb{N}$, the function $\varphi_{n}(t)=\left\|u(t)-\mathrm{v}_{n}\right\|^{2}$ belongs to $L_{\text {loc }}^{1}[0, \infty)$, so by the Lebesgue differentiation theorem

$$
\lim _{\tau \rightarrow 0} \frac{1}{\tau} \int_{0}^{\tau} \varphi_{n}(t+\sigma) d \sigma=\varphi_{n}(t)
$$

holds for a.e. $t \geq 0$. It follows that there is a set $E \subset[0, \infty)$ whose complement has measure zero and such that (6.2) holds for any $t \in E$ and any $n \in \mathbb{N}$. We show that (6.1) holds for any $t \in E$. For any $t \geq 0$ and any $\tau>0$ we have, 
by the triangle inequality in $L^{2}([0, \tau], U)$,

$$
\begin{aligned}
\left(\frac{1}{\tau} \int_{0}^{\tau}\|u(t+\sigma)-u(t)\|^{2} d \sigma\right)^{1 / 2} & \\
& \leq\left(\frac{1}{\tau} \int_{0}^{\tau}\left\|u(t+\sigma)-\mathrm{v}_{n}\right\|^{2} d \sigma\right)^{1 / 2}+\left\|\mathrm{v}_{n}-u(t)\right\| \\
& =\left[\left(\frac{1}{\tau} \int_{0}^{\tau} \varphi_{n}(t+\sigma) d \sigma\right)^{1 / 2}-\left(\varphi_{n}(t)\right)^{1 / 2}\right]+2\left\|\mathrm{v}_{n}-u(t)\right\|
\end{aligned}
$$

Let $t \in E$ be fixed. For any $\varepsilon>0$ there is an $n \in \mathbb{N}$ such that the second term on the right-hand side is $\leq \varepsilon$. For this $n$, the first term on the right-hand side is $\leq \varepsilon$ for $\tau$ sufficiently small, so the left-hand side is $\leq 2 \varepsilon$, which proves (6.1).

The above proof follows the idea of the proof of a related result in Diestel and Uhl [7, p. 49]. In [18], Theorem 2.3 was obtained as a consequence of (2.16). Now it seems to me more natural to prove Theorem 2.3 directly (and then (2.16) becomes an easy consequence, as explained in $\S 2$ ).

Proof of Theorem 2.3. As already mentioned in $\S 2$, the fact that $x$ is the unique strong solution of $(1.2 \mathrm{a})$ follows from the representation (2.5). The details are in Weiss [16, Theorem 3.9] and we do not repeat them here. Our aim is to prove that $(1.2 \mathrm{~b})$ holds for a.e. $t \geq 0$.

Both $u$ and $y$ are equivalence classes of functions modulo equality a.e. We choose one representative for $u$ and one for $y$, and for the rest of this proof we consider $u$ and $y$ to be well defined in every point $t \geq 0$. Let $\mathscr{T}$ be the set of points $t \in[0, \infty)$ where the following two conditions are satisfied:

(i) $y$ has a Lebesgue point at $t$ and

$$
y(t)=\lim _{\tau \rightarrow 0} \frac{1}{\tau} \int_{0}^{\tau} y(t+\sigma) d \sigma,
$$

(ii) the equality (6.1) holds.

By a well-known theorem on Lebesgue points (see Diestel and Uhl [7, p. 49]) and by Lemma 6.1, almost every $t \geq 0$ belongs to $\mathscr{T}$. We will show that $x(t) \in D\left(C_{L}\right)$ and $(1.2 \mathrm{~b})$ hold for any $t \in \mathscr{T}$.

We need formula (4.8) from the proof of Lemma 4.4, so we recall the notation used there. For any $\tau>0$, the operators $C_{L}^{\tau} \in \mathscr{L}(X, Y)$ and $D^{\tau} \in \mathscr{L}(U, Y)$ are defined by (4.3) and (4.4), and for any $t \geq 0$, the function $\varepsilon_{t} \in L_{\mathrm{loc}}^{2}([0, \infty), U)$ is defined by (4.7). Then (by the same argument as in the proof of Lemma 4.4) formula (4.8) holds.

We show that for any $t \in \mathscr{T}$, each term in (4.8) has a limit as $\tau \rightarrow 0$. Assume $t \in \mathscr{T}$. By condition (i) above, the left-hand side of (4.8) tends to $y(t)$ as $\tau \rightarrow 0$. By (2.14) (the regularity assumption), $\lim _{\tau \rightarrow 0} D^{\tau} u(t)=D u(t)$. We have seen in the proof of Lemma 4.4 that for any $\tau \in(0,1]$

$$
\left\|\frac{1}{\tau} \int_{0}^{\tau}\left(\mathbb{F}_{\infty} \varepsilon_{t}\right)(\sigma) d \sigma\right\| \leq\left\|\mathbb{F}_{1}\right\|\left(\frac{1}{\tau} \int_{0}^{\tau}\left\|\varepsilon_{t}(\sigma)\right\|^{2} d \sigma\right)^{1 / 2} .
$$

This, together with condition (ii) above shows that the last term on the righthand side of (4.8) tends to 0 as $\tau \rightarrow 0$. It follows that the term $C_{L}^{\tau} x(t)$ in (4.8) also must have a limit as $\tau \rightarrow 0$. 
By the definitions of $C_{L}^{\tau}$ and $C_{L}$ (see (4.3) and (2.12)) this means that $x(t) \in D\left(C_{L}\right)$ and $\lim _{\tau \rightarrow 0} C_{L}^{\tau} x(t)=C_{L} x(t)$. Now we know the limit of each term in (4.8) and we can write down the equality obtained by taking limits: it is exactly $(1.2 \mathrm{~b})$.

Remark 6.2. With the notation of Theorem 2.3, if $t \geq 0$ is such that both $u$ and $y$ are continuous from the right at $t$, then $x(t) \in D\left(C_{L}\right)$ and $y(t)=$ $C_{L} x(t)+D u(t)$. Indeed, it is clear that both conditions (i) and (ii) of the preceding proof are satisfied at $t$. This fact is useful in the analysis of feedback systems (as will be discussed in another article).

Concerning extensions to Banach spaces and functions of class $L_{\mathrm{loc}}^{p}$, see Remark 2.4.

\section{REFERENCES}

1. W. Arendt and J. Prüss, Vector-valued Tauberian theorems and asymptotic behavior of linear Volterra equations, SIAM J. Math. Anal. 23 (1992), 412-448.

2. C. J. K. Batty, Tauberian theorems for the Laplace-Stieltjes transform, Trans. Amer. Math. Soc. 322 (1990), 783-804.

3. R. F. Curtain, Equivalence of input-output stability and exponential stability, Part II, Systems and Control Letters 12 (1989), 235-239.

4. ical Systems Theory (H. Nijmeijer and J. M. Schumacher, eds.), Springer-Verlag, Berlin, 1989, pp. 101-128.

5. R. F. Curtain, H. Logemann, S. Towniey, and H. Zwart, Well-posedness, stabilizability and admissibility for Pritchard-Salamon systems, Institut für Dynamische Systeme, Universität Bremen, Report \#260, 1992.

6. R. F. Curtain and G. Weiss, Well posedness of triples of operators (in the sense of linear systems theory), Control and Estimation of Distributed Parameter Systems (F. Kappel, K. Kunisch, and W. Schappacher, eds.), Birkhäuser, Basel, 1989, pp. 41-59.

7. J. Diestel and J. J. Uhl, Vector measures, Math. Surveys, vol. 15, Amer. Math. Soc., Providence, RI, 1977.

8. G. Doetsch, Handbuch der Laplace-Transformation, Bd. I, Birkhäuser Verlag, Basel, 1950.

9. Y. Fourés and I. E. Segal, Causality and analyticity, Trans. Amer. Math. Soc. 78 (1955), 385-405.

10. P. A. Fuhrmann, Linear systems and operators in Hilbert space, McGraw-Hill, New York, 1981.

11. G. Greiner, J. Voigt, and M. Wolff, On the spectral bound of the generator of semigroups of positive operators, J. Operator Theory 5 (1981), 245-256.

12. J. W. Helton, Systems with infinite-dimensional state space: the Hilbert space approach, Proc. IEEE 64 (1976), 145-160.

13. R. Rebarber, Conditions for the equivalence of internal and external stability for distributed parameter systems, IEEE Trans. Automat. Control 38 (1993), 994-998.

14. D. Salamon, Infinite dimensional systems with unbounded control and observation: a functional analytic approach, Trans. Amer. Math. Soc. 300 (1987), 383-431.

15. __ Realization theory in Hilbert space, Math. Systems Theory 21 (1989), 147-164.

16. G. Weiss, Admissibility of unbounded control operators, SIAM J. Control Optim. 27 (1989), 527-545.

17. $\underset{17-43}{ }$, Admissible observation operators for linear semigroups, Israel J. Math. 65 (1989),

, The representation of regular linear systems on Hilbert spaces, Control and Estimation of Distributed Parameter Systems (F. Kappel, K. Kunisch, and W. Schappacher, eds.), Birkhäuser, Basel, 1989, pp. 401-416. 
19. __ Representation of shift invariant operators on $L^{2}$ by $H^{\infty}$ transfer functions: an elementary proof, a generalization to $L^{p}$ and a counterexample for $L^{\infty}$, Math. Control Signals Systems 4 (1991), 193-203.

20. H. Zwart, Private communication, 1988.

Department of Theoretical Mathematics, The Weizmann Institute of Science, 76100 REHOVOT, ISRAEL

Current address: Department of Electrical Engineering, Ben-Gurion Univerisy, 84105 Beer Sheva, Israel

E-mail address: weiss@bguvm.bgu.ac.il 